\title{
Reallocation of an infinitely divisible good
}

Citation for published version (APA):

Klaus, B. E., Storcken, A. J. A., \& Peters, H. J. M. (1995). Reallocation of an infinitely divisible good. METEOR, Maastricht University School of Business and Economics. METEOR Research Memorandum No. 012 https://doi.org/10.26481/umamet.1995012

Document status and date:

Published: 01/01/1995

DOI:

10.26481/umamet.1995012

Document Version:

Publisher's PDF, also known as Version of record

\section{Please check the document version of this publication:}

- A submitted manuscript is the version of the article upon submission and before peer-review. There can be important differences between the submitted version and the official published version of record.

People interested in the research are advised to contact the author for the final version of the publication, or visit the DOI to the publisher's website.

- The final author version and the galley proof are versions of the publication after peer review.

- The final published version features the final layout of the paper including the volume, issue and page numbers.

Link to publication

\footnotetext{
General rights rights.

- You may freely distribute the URL identifying the publication in the public portal. please follow below link for the End User Agreement:

www.umlib.nl/taverne-license

Take down policy

If you believe that this document breaches copyright please contact us at:

repository@maastrichtuniversity.nl

providing details and we will investigate your claim.
}

Copyright and moral rights for the publications made accessible in the public portal are retained by the authors and/or other copyright owners and it is a condition of accessing publications that users recognise and abide by the legal requirements associated with these

- Users may download and print one copy of any publication from the public portal for the purpose of private study or research.

- You may not further distribute the material or use it for any profit-making activity or commercial gain

If the publication is distributed under the terms of Article $25 \mathrm{fa}$ of the Dutch Copyright Act, indicated by the "Taverne" license above, 


\title{
REALLOCATION OF AN INFINITELY DIVISIBLE GOOD
}

\author{
B. KLAUS, H. PETERS, AND T. STORCKEN
}

\begin{abstract}
We consider the problem of reallocating the total initial endowments of an infinitely divisible commodity among agents with single-peaked preferences. With the uniform reallocation rule we propose a solution which satisfies many appealing properties, describing the effect of population and endowment variations on the outcome. The central properties which are studied in this context are population monotonicity, bilateral consistency, (endowment) monotonicity and (endowment) strategy-proofness. Furthermore, the uniform reallocation rule is Pareto optimal and satisfies several equity conditions, e.g., equaltreatment and envy-freeness. We study the trade-off between properties concerning variation and properties concerning equity. Furthermore, we provide several characterizations of the uniform reallocation rule based on these properties.
\end{abstract}

\section{INTRODUCTION}

In this paper we study situations where the total of initial endowments of an infinitely divisible good is reallocated among a group of agents. In many cases where free disposal of the good is not allowed (non-price models) it is natural to assume that the agents' preferences over their shares of the good are single-peaked. Each agent has an optimal share of the good, below which and above which preference is decreasing.

There is a wide literature exploring the situation where the problem is reduced to the allocation of a total endowment. As described in Sprumont [7], rationing in a two-good economy in which prices are in disequilibrium can be interpreted as such a distribution problem with total endowment. A solution for this class of problems satisfying many appealing properties is the uniform rule. Benassy [2] described the uniform rule as a strategy-proof rationing scheme: an agent who misrepresents his preference cannot improve his outcome. Sprumont [7] started the axiomatic analysis in 1991. He proved that the uniform rule is the only rule which satisfies Pareto optimality, strategy-proofness and anonymity. Ching [3] weakens anonymity to a condition called equal treatment of equals: agents announcing the same preferences are treated equally. The axiomatic analysis of Thomson (see [8],

Date. August, 1995. 
[9] and [10]) provides several characterizations of the uniform rule including consistency and monotonicity properties.

In this paper we study similar properties in the more general setting of economies where agents have initial endowments. This extension of the model quite naturally arises if we observe distribution problems with total endowments where preferences might change over time. Consider for example the distribution of a task (e.g., fixed amount of teaching hours) among the members of a group. The (single-peaked) preferences of the agents do not only depend on the total endowment, but also on external factors (time for research, other tasks) which are not fixed. So, over time, preferences might change, calling for a reallocation of the task.

Another interpretation of the model can be found in a recent paper of Barbera, Jackson and Neme [1]. There, sharing problems where agents might have natural claims, or are treated with different priorities, are studied. In this setting they characterize the class of distribution rules that are strategy-proof and Pareto optimal, but which allow for an asymmetric treatment of the agents. Adding a third condition, describing a kind of individual monotonicity, yields a subclass of strategy-proof and Pareto optimal rules which they call sequential allotment rules and which they consider to be a natural extension of the procedure which underlies the uniform rule. By applying uniform division in the stepwise definition of a sequential allotment rule, thereby reducing the computation to one step, the uniform reallocation rule, introduced in Klaus, Peters and Storcken [6], is obtained.

In Klaus, Peters and Storcken [6] the main result is the characterization of the uniform reallocation rule by Pareto optimality, strategy-proofness and an equal-treatment condition based on the preferences and the net demands of the agents. This equity condition, which corresponds to Ching's equaltreatment condition for division problems, may be replaced by anonymity and translation invariance.

Like the uniform rule, the uniform reallocation rule satisfies many desirable properties, which we study in the sequel. We can strengthen equaltreatment to envy-freeness which, in our setting, is formalized in terms of allotment changes and not in terms of the outcome as in the case of dividing a total endowment. Our first result (Theorem 3.1) is that, similar to the total endowment case (see Thomson [10], Lemma 1), the uniform reallocation rule is the unique reallocation rule satisfying Pareto optimality, peaks-onliness and envy-freeness.

Besides strategy-proofness, which describes the influence of certain preference variations on the outcome, and Pareto optimality, the uniform reallocation rule has several properties incorporating the variation of the remaining model assumptions.

One such property, introduced by Thomson [8] for the total endowment case, is population monotonicity. In the reallocation case this property describes the impact of merging two reallocation problems. We show (The- 
orem 3.2) that in the characterization of Theorem 3.1 peaks-onliness can be replaced by population monotonicity.

A further monotonicity property, endowment monotonicity, describes the change of the solution if certain endowment variations are considered. By decreasing (increasing) the endowments in case of excess demand (supply), no individual is better off than before. This monotonicity condition is an extension of the one-sided resource-monotonicity of Thomson [10], introduced for division problems. Thomson proves ([10], Theorem 2) that the uniform rule is the only rule satisfying Pareto optimality, envy-freeness and one-sided resource-monotonicity for a restricted domain of single-peaked, continuous preferences. For the reallocation case a similar result (Theorem 4.4) can be deduced where the conditions of envy-freeness and monotonicity are adapted as indicated above. However, the proof of this characterization of the uniform reallocation rule is based on a different argument and remains valid for the whole domain of single-peaked preferences.

The next property of the uniform reallocation rule we study is bilateral consistency. For the total endowment case, bilateral consistency of a rule requires the following. Consider a division assigned by a rule and assume that all agents except two leave with their assigned quantities of the good. If the remaining agents divide the remaining endowment again by applying the same rule, then they receive the same shares as before. In Thomson [9] two characterizations of the uniform rule by means of Pareto optimality, bilateral consistency and continuity in the total amount to divide are provided. In the first characterization ([9], Theorem 1) envy-freeness singles out the uniform rule. In the second characterization ([9], Theorem 2) envy-freeness is replaced by individual rationality from equal division: no agent, after the distribution, is worse off than in the case of equally dividing the total endowment. In a recent study, Dagan shows that the continuity property may be skipped (see [4], Theorem 2 and Theorem 3).

In reallocation situations bilateral consistency not only prescribes the indifference of the outcome to the splitting off of a group in a certain way, but it also includes an equity component. The leftover of the departing agents is, up to domain restrictions, equally added to the endowments of the remaining agents. Individual rationality from equal division in the total endowment case corresponds quite naturally to individual rationality (with respect to the initial endowments) in reallocation situations. After the reallocation no agent is worse off. The latter two conditions (bilateral consistency and individual rationality) together with Pareto optimality determine the uniform reallocation rule for reallocation problems with at least three agents (Theorem 5.2). For reallocation problems with at least four agents individual rationality can be replaced by envy-freeness (Theorem 5.3). The proofs of Dagan's characterizations ([4], Theorem 2 and Theorem 3) can be adapted to the reallocation case. This yields different proofs of Theorem 5.2 and Theorem 5.3 for problems with at least four agents. Further characteriza- 
tions of the uniform reallocation rule can be obtained by Pareto optimality, bilateral consistency and extra conditions, for instance boundedness (of the outcome) by endowments and peaks (Theorem 5.1).

Finally, endowment strategy-proofness is studied. If initial endowments are private information it might happen that agents manipulate the outcome by only reporting - showing - a smaller part of their endowments. Reallocation rules where agents cannot profit from withholding parts of their endowments are called endowment strategy-proof. Endowment strategyproofness together with Pareto optimality, bilateral consistency and the dummy property (agents who have their peak as initial endowment do not participate in the reallocation) characterizes the uniform reallocation rule (Theorem 6.3). In Theorem 6.4 we show that we can replace endowment strategy-proofness and the dummy property by equal-treatment and a property called reversibility. This latter condition links the outcomes of excess demand and excess supply. To be more precise, consider a situation with demanders, having their peaks above their initial endowments, and suppliers, having their peaks below their initial endowments. Now this situation is reversed by turning demanders into suppliers with supply equal to their former demand and suppliers into demanders in a similar way. Reversibility requires that the allotment changes of the latter problem are opposite to those of the former.

The paper proceeds as follows. In Section 2 we introduce the model and the uniform reallocation rule. In Sections 3, 4, 5 and 6 we introduce the equity and variation properties which yield several characterizations of the uniform reallocation rule. An overview over the results is given in Section 7 and the independence of the axioms used in the characterizations is shown. Furthermore, a discussion of the sensitivity of the model assumptions is included.

\section{Reallocations}

Consider exchange economies with a single good for which the agents have single-peaked (ordinal) utility, for instance strictly concave utility functions with a global optimum. So, the commodity space is one dimensional: $I R_{+}$. Let $i$ be an agent. Then his utility function $u_{i}$ is a continuous function from $\mathbb{R}_{+}$to $\mathbb{R}$ such that

- there is a unique point $\hat{u}_{i}$ at which $u_{i}$ is maximal

- for all $\alpha, \beta \in \mathbb{R}_{+}, u_{i}(\alpha)<u_{i}(\beta)$ if $\alpha<\beta \leq \hat{u}_{i}$ or if $\alpha>\beta \geq \hat{u}_{i}$.

The point $\hat{u}_{i}$ is called the peak of $i$. Denote the set of all these utility functions by $\mathcal{U}$. A set of agents is denoted by $N \subset \mathbb{N}$. Furthermore, $\mathcal{U}^{N}$ denotes the set of $N$-tuples $u$ of utility functions. So, $u=\left\langle u_{i}\right\rangle_{i \in N}$, where $u_{i}$ is the utility function of agent $i$. If $u, v \in \mathcal{U}^{N}$, then $\hat{u}=\hat{v}$ indicates that 
for every agent $i$ the peak at $u\left(\hat{u}_{i}\right)$ is equal to that at $v\left(\hat{v}_{i}\right)$.

A reallocation problem or short a problem is a triple $\langle N, e, u\rangle$, where $N$ is a nonempty and finite set of agents, $N \subset \mathbb{I N}, e$ is a vector of initial endowments $e=\left\langle e_{i}\right\rangle_{i \in N} \in \mathbb{R}_{+}^{N}$ and $u$ is a profile of utility functions: $u \in$ $\mathcal{U}^{N}$. At $e$ the initial endowment of agent $i$ is $e_{i}$.

In problem $\langle N, e, u\rangle$ agent $i$ is a demander whenever his endowment $e_{i}$ is strictly less than his peak $\hat{u}_{i}$. In that case he wants more of the good. His (net) demand is denoted by $d_{i}(N, e, u)$, so $d_{i}(N, e, u):=\hat{u}_{i}-e_{i}$. Denote the set of demanders by $D(N, e, u)$. A supplier is an agent $j$ who has an endowment that is strictly greater than his peak. The supply of such an agent is $s_{j}(N, e, u):=e_{j}-\hat{u}_{j}$. The set of suppliers is denoted by $S(N, e, u)$. If agent $k$ is neither a supplier nor a demander, then his peak $\hat{u}_{k}$ equals his endowment $e_{k}$. In that case he favors no trade, and is called a nontrader. Let $d(N, e, u):=\sum_{i \in D(N, e, u)} d_{i}(N, e, u)$ denote total demand and $s(N, e, u):=\sum_{i \in S(N, e, u)} s_{i}(N, e, u)$ total supply. The excess demand function $z(N, e, u):=d(N, e, u)-s(N, e, u)$ may be positive, zero, or negative. If it is positive we say that the problem has excess demand. If it is zero, the problem is balanced and one would expect that the reallocation is such that every agent gets his peak. If it is negative, then we have excess supply.

A vector $x=\left\langle x_{i}\right\rangle_{i \in N} \in \mathbb{R}_{+}^{N}$ is called feasible (at problem $\langle N, e, u\rangle$ ) or a reallocation if $\sum_{i \in N} x_{i}=\sum_{i \in N} e_{i}$. A reallocation $x$ is called Pareto optimal (at problem $\langle N, e, u\rangle$ ), if there is no reallocation $y=\left\langle y_{i}\right\rangle_{i \in N}$ in $I R_{+}^{N}$, such that

$$
\begin{aligned}
u_{i}\left(x_{i}\right) & \leq u_{i}\left(y_{i}\right) \text { for all agents } i \in N \text { and } \\
u_{j}\left(x_{j}\right) & <u_{j}\left(y_{j}\right) \text { for at least one agent } j \in N .
\end{aligned}
$$

Utility strictly increases, if the peak is approached from above or from below. Therefore, a reallocation $x \in \mathbb{R}_{+}^{N}$ is Pareto optimal at problem $\langle N, e, u\rangle$, precisely when $x$ is same-sided, i.e., $x_{i} \leq \hat{u}_{i}$ for all $i \in N$ or $x_{i} \geq \hat{u}_{i}$ for all $i \in N$. Consequently, a reallocation $x \in \mathbb{R}_{+}^{N}$ is Pareto optimal if and only if,

$$
\begin{aligned}
& x_{i} \leq \hat{u}_{i} \text { for all } i \in N \text { whenever } z(N, e, u)>0 \quad \text { (excess demand), } \\
& x_{i} \geq \hat{u}_{i} \text { for all } i \in N \text { whenever } z(N, e, u)<0 \text { (excess supply), and } \\
& x_{i}=\hat{u}_{i} \text { for all } i \in N \text { whenever } z(N, e, u)=0 \quad \text { (balancedness). }
\end{aligned}
$$

Sprumont [7] uses same-sidedness as definition of Pareto optimality.

In several properties, discussed hereafter, the number of agents is not fixed, therefore solutions will be defined over the set of all problems. To avoid repetition of the Pareto optimality condition, it is incorporated in the definition of a rule as follows.

A pre-rule $\psi$ assigns to every problem $\langle N, e, u\rangle$ a reallocation $\psi(N, e, u)$. 
A rule $\varphi$ is a Pareto optimal pre-rule.

Let $\langle N, e, u\rangle$ be a problem and $i \in N$. Then $\varphi_{i}(N, e, u)$ denotes the allotment of $i$ under rule $\varphi$ at problem $\langle N, e, u\rangle$. Furthermore, $\triangle \varphi_{i}(N, e, u):=$ $\varphi_{i}(N, e, u)-e_{i}$ denotes the actual allotment change for agent $i$ under $\varphi$ at $\langle N, e, u\rangle$. If $N$ is fixed, then we write $\varphi(e, u), \varphi_{i}(e, u), \triangle \varphi(e, u), \triangle \varphi_{i}(e, u)$ instead of $\varphi(N, e, u), \varphi_{i}(N, e, u), \triangle \varphi(N, e, u)$ or $\triangle \varphi_{i}(N, e, u)$ respectively. We adopt analogous conventions for the endowment vector $e$ and the profile of utility functions $u$.

An agent $i$ is non-satiated under pre-rule $\psi$ at problem $\langle N, e, u\rangle$ if $\widehat{u}_{i} \neq$ $\psi_{i}(N, e, u)$.

A special rule is the uniform reallocation rule $U^{r}$, introduced in [6]. For a problem $\langle N, e, u\rangle$ it is defined as follows,

$$
U_{j}^{r}(N, e, u):=\left\{\begin{array}{lll}
\min \left\{\hat{u}_{j}, e_{j}+\lambda\right\} & \text { if } z(N, e, u)>0 & \text { (excess demand) } \\
\hat{u}_{j} & \text { if } z(N, e, u)=0 & \text { (balancedness) } \\
\max \left\{\hat{u}_{j}, e_{j}-\lambda\right\} & \text { if } z(N, e, u)<0 & \text { (excess supply) }
\end{array}\right.
$$

for every $j \in N$, where $\lambda \geq 0$ solves $\sum_{i \in N} U_{i}^{r}(N, e, u)=\sum_{i \in N} e_{i}$.

So, if there is excess demand, then all suppliers and non-traders get their peaks. Demanders either receive their peaks or get maximal equal allotment change $\lambda$. In excess supply all non-satiated agents get minimal allotment change $-\lambda$. Hence, agents are either satiated or receive the same (maximal or minimal) allotment change. In fact, combined with Pareto optimality this exactly determines the uniform reallocation rule:

Lemma 2.1. The uniform reallocation rule is the only rule $\psi$ such that, for every problem $\langle N, e, u\rangle$ non-satiated agents

(a) obtain maximal allotment change $\max \left\{\Delta \psi_{i}(N, e, u) \mid i \in N\right\}$ if the problem is of excess demand,

(b) obtain minimal allotment change $\min \left\{\Delta \psi_{i}(N, e, u) \mid i \in N\right\}$ if the problem is of excess supply,

(c) do not exist, if the problem is balanced, i.e., all agents get their peaks.

Proof. By definition, the uniform reallocation rule is same-sided and therefore Pareto optimal. Hence, the uniform reallocation rule is actually a rule. Also by definition, $U^{r}$ satisfies (a), (b), and (c). Suppose $\varphi$ is a rule satisfying (a), (b), and (c), and let $\langle N, e, u\rangle$ be a problem. We prove that $\varphi(N, e, u)=U^{r}(N, e, u)$. If the problem is balanced then this is obvious, so we suppose that the problem has excess demand (the excess supply case is analogous). Hence, $z(N, e, u)>0$.

If $e_{i}>\varphi_{i}(N, e, u)$ for some $i \in N$, then by feasibility there is a $j \in N$ such that $e_{j}<\varphi_{j}(N, e, u)$. Hence $\Delta \varphi_{i}(N, e, u)$ is not maximal, so by (a) it follows that in that case $\varphi_{i}(N, e, u)=\widehat{u}_{i}$.

So, if $i$ is not satiated, then $\Delta \varphi_{i}(N, e, u) \geq 0$. Because of same-sidedness we have $\widehat{u}_{i} \geq \varphi_{i}(N, e, u)$ for every $i \in N$.

For suppliers $i$, where $e_{i}>\widehat{u}_{i}$, this means that $\widehat{u}_{i}=\varphi_{i}(N, e, u)$. 
For non-traders $i$, where $e_{i}=\widehat{u}_{i}$, this also means that $\widehat{u}_{i}=\varphi_{i}(N, e, u)$.

So, only demanders can be non-satiated, in which case they obtain maximal allotment change. Because of same-sidedness, it follows that nonsatiated demanders obtain less than their peaks. But then it follows that $\varphi(N, e, u)=U^{r}(N, e, u)$.

\section{PEAKS-ONLY RULES}

In this section we focus on rules which base the outcomes on the peaks instead of the complete utility functions. The uniform reallocation rule is such a rule. Moreover, it will appear to be the only rule which satisfies this condition and at which no agent envies another one. Furthermore, a monotonicity condition is discussed. It is shown that this condition and envy-freeness imply the peaks-onliness condition. Because the uniform reallocation rule satisfies this condition, this implication yields a second characterization of this rule.

Let $\varphi$ be a pre-rule. Then $\varphi$ is said to be peaks-only if for all problems $\langle N, e, u\rangle$ and $\left\langle N^{\prime}, e^{\prime}, u^{\prime}\right\rangle$, with $N=N^{\prime}, e=e^{\prime}$ and $\hat{u}=\hat{u}^{\prime}$,

$$
\varphi(N, e, u)=\varphi\left(N^{\prime}, e^{\prime}, u^{\prime}\right) \text {. }
$$

So, a rule $\varphi$ is peaks-only if, and only if, the outcomes only depend on the peaks of the utility functions and not on the whole functions. As a manner of speaking, peaks-only rules ignore intensities. Nevertheless, many well-known rules are peaks-only. The uniform rule, the proportional rule, equal division and hierarchical rules, rules which are discussed in Section 7, are peaks-only. It is evident that by its definition the uniform reallocation rule is also peaks-only. Clearly, if a rule takes intensities into account, then it is apt to be vulnerable to strategic behavior and more difficult to apply.

The pre-rule $\varphi$ is said to be envy-free if for all problems $\langle N, e, u\rangle$ and all individuals $i, j \in N$ with $\Delta \varphi_{j}(N, e, u)+e_{i} \geq 0$,

$$
u_{i}\left(\Delta \varphi_{j}(N, e, u)+e_{i}\right) \leq u_{i}\left(\varphi_{i}(N, e, u)\right)
$$

So, $i$ envies $j$ if $i$ prefers $j$ 's allotment change, added to his endowment, to his own allotment-provided the former is feasible. The uniform reallocation rule is envy-free. For instance, in case of excess demand, only demanders can be non-satiated and, if so, they obtain the same, maximal allotment change.

The well-known property of envy-freeness was introduced by Foley [5] for resource allocation problems. Envy-freeness for division problems with single-peaked preferences was first used by Sprumont in his axiomatic analysis of the uniform rule, [7].

The following theorem characterizes the uniform reallocation rule as the only rule which is envy-free and peaks-only. ${ }^{1}$ The main idea of the proof

\footnotetext{
${ }^{1}$ Here as well as elsewhere in the paper, the expression "characterization" implies the logical independence of the characterizing axioms. For all characterizations appearing
} 
is that, because of these two properties, allotment changes for non-satiated agents are maximal or minimal, depending on whether the problem is in excess demand or in excess supply. As this typically describes the uniform reallocation rule, we are done.

The theorem and its proof are similar to Lemma 3 and its proof in Thomson [10], which treats the case of total endowment instead of initial endowments.

Theorem 3.1. The uniform reallocation rule is the only envy-free and peaksonly rule.

Proof. Clearly, the uniform reallocation rule is an envy-free and peaks-only rule. In order to prove that it is the only one let $\varphi$ be an envy-free and peaks-only rule. Let $\langle N, e, u\rangle$ be a problem. We prove that $\varphi(N, e, u)=$ $U^{r}(N, e, u)$. By Pareto optimality, it follows immediately that $\varphi(N, e, u)=$ $U^{r}(N, e, u)=\hat{u}$ if $z(N, e, u)=0$ (balancedness). Without loss of generality suppose that $z(N, e, u)>0$ (excess demand). By Lemma 2.1 it is sufficient to prove that non-satiated agents get maximal allotment changes at $\varphi$. Let $i \in N$ be a non-satiated agent at $\varphi(N, e, u)$. Hence, by samesidedness, $\varphi_{i}(N, e, u)<\hat{u}_{i}$. Consider the allotment change $\triangle \varphi_{j}(N, e, u)$ of an arbitrary agent $j$. As there is a utility profile $v \in \mathcal{U}^{N}$, such that $\hat{v}=\hat{u}$ and $v_{i}(x)>v_{i}\left(\varphi_{i}(N, e, u)\right)$ for all $x>\varphi_{i}(N, e, u)$, it follows by envyfreeness and peaks-onliness that $\triangle \varphi_{j}(N, e, u)+e_{i} \leq \varphi_{i}(N, e, u)$. Hence, $\triangle \varphi_{j}(N, e, u) \leq \triangle \varphi_{i}(N, e, u)$.

The following characterization of the uniform reallocation rule involves population monotonicity. Loosely speaking, a rule is population monotonic if merging two disjoint problems either both of excess demand or both of excess supply, makes in one subgroup either all agents weakly better off or all agents weakly worse off. So, if we add a demander to a problem $\langle N, e, u\rangle$ with excess demand, yielding problem $\left\langle N^{\prime}, \epsilon^{\prime}, u^{\prime}\right\rangle$, then either all agents in $N$ weakly prefer the outcome at $\langle N, e, u\rangle$ to that at $\left\langle N^{\prime}, e^{\prime}, u^{\prime}\right\rangle$ or all agents in $N$ prefer it the other way around.

A pre-rule $\varphi$ is said to be population monotonic, if for all problems $\langle N, e, u\rangle$ and $\left\langle N^{\prime}, e^{\prime}, u^{\prime}\right\rangle$, such that $z(N, e, u) \cdot z\left(N^{\prime}, e^{\prime}, u^{\prime}\right)>0$ and $N \cap N^{\prime}=\emptyset$,

$$
\begin{array}{ll}
\text { either } & u_{i}\left(\varphi_{i}(N, e, u)\right) \geq u_{i}\left(\varphi_{i}\left(N \cup N^{\prime},\left\langle e, e^{\prime}\right\rangle,\left\langle u, u^{\prime}\right\rangle\right)\right) \text { for all } i \in N \\
\text { or } & u_{i}\left(\varphi_{i}(N, e, u)\right) \leq u_{i}\left(\varphi_{i}\left(N \cup N^{\prime},\left\langle e, e^{\prime}\right\rangle,\left\langle u, u^{\prime}\right\rangle\right)\right) \text { for all } i \in N .
\end{array}
$$

Here $\left\langle e, e^{\prime}\right\rangle$ is the vector $x$ in $\mathbb{R}_{+}^{N \cup N^{\prime}}$ such that $x_{i}=e_{i}$ for all $i \in N$ and $x_{i}=e_{i}^{\prime}$ for all $i \in N^{\prime}$. The profile $\left\langle u, u^{\prime}\right\rangle \in \mathcal{U}^{N \cup N^{\prime}}$ has a similar meaning.

Note that $z(N, e, u) \cdot z\left(N^{\prime}, e^{\prime}, u^{\prime}\right)>0$ if, and only if, both problems have

in this paper, however, the proof of logical independence of the axioms is postponed to Section 7 . 
excess demand or both problems have excess supply.

The uniform reallocation rule is population monotonic. To see this, let $\langle N, e, u\rangle$ and $\left\langle N^{\prime}, e^{\prime}, u^{\prime}\right\rangle$ be two problems with excess demand. Let $\lambda, \lambda^{\prime}$ and $\mu$ be the maximal allotment changes of $U^{r}$ in the problems $\langle N, e, u\rangle$, $\left\langle N^{\prime}, e^{\prime}, u^{\prime}\right\rangle$ and $\left\langle N \cup N^{\prime},\left\langle e, e^{\prime}\right\rangle,\left\langle u, u^{\prime}\right\rangle\right\rangle$ respectively. Let $i, j \in N$. Suppose $i$ is strictly better off at $\langle N, e, u\rangle$ than at $\left\langle N \cup N^{\prime},\left\langle e, e^{\prime}\right\rangle,\left\langle u, u^{\prime}\right\rangle\right\rangle$. Suppose for $j$ the converse holds. Then $i$ and $j$ must be demanders. Hence, the agents $i$ and $j$ are not satiated at problem $\left\langle N \cup N^{\prime},\left\langle e, e^{\prime}\right\rangle,\left\langle u, u^{\prime}\right\rangle\right\rangle$ and $\langle N, e, u\rangle$ respectively. So,

$$
\begin{aligned}
\mu & =\triangle \varphi_{i}\left(N \cup N^{\prime},\left\langle e, e^{\prime}\right\rangle,\left\langle u, u^{\prime}\right\rangle\right)<\triangle \varphi_{i}(N, e, u) \leq \lambda \text { and } \\
\lambda & =\triangle \varphi_{j}(N, e, u)<\triangle \varphi_{j}\left(N \cup N^{\prime},\left\langle e, e^{\prime}\right\rangle,\left\langle u, u^{\prime}\right\rangle\right) \leq \mu .
\end{aligned}
$$

Because this cannot be true, all agents in $N$ are either weakly better off in $\langle N, e, u\rangle$ than in $\left\langle N \cup N^{\prime},\left\langle e, e^{\prime}\right\rangle,\left\langle u, u^{\prime}\right\rangle\right.$, or all are weakly worse off. The proof for problems with excess supply is similar.

Hence, the uniform reallocation rule is population monotonic. Moreover, the following theorem shows that envy-freeness and population monotonicity characterize the uniform reallocation rule.

Theorem 3.2. The uniform reallocation rule is the only envy-free and population monotonic mule.

Proof. In order to prove that $U^{r}$ is the only envy-free and population monotonic rule suppose $\varphi$ is such a rule. It is sufficient to prove that $\varphi$ is peaksonly. Let $\langle N, e, u\rangle$ be a problem and $v \in \mathcal{U}^{N}$ such that $\hat{u}=\hat{v}$. Without loss of generality let $N=\{1,2, \ldots, n\}$ and suppose $z(N, e, u)>0$. Consider $N^{\prime}=\{n+1, \ldots, 2 n\}$. Take $e^{\prime} \in \mathbb{I}^{N^{\prime}}$ such that $e_{i+n}^{\prime}=e_{i}$ for all $i \in N$. Let $u^{\prime} \in \mathcal{U}^{N^{\prime}}$ be such that $u_{i+n}^{\prime}=v_{i}$ for all $i \in N$. It is sufficient to show that $\varphi_{i}(N, e, u)=\varphi_{i+n}\left(N^{\prime}, e^{\prime}, u^{\prime}\right)$, because this also implies $\varphi_{i}(N, e, v)=\varphi_{i+n}\left(N^{\prime}, e^{\prime}, u^{\prime}\right)$. Clearly, $z\left(N^{\prime}, e^{\prime}, u^{\prime}\right)>0$. Consider $\left\langle N \cup N^{\prime},\left\langle e, e^{\prime}\right\rangle,\left\langle u, u^{\prime}\right\rangle\right\rangle$. Then $z\left(N \cup N^{\prime},\left\langle e, e^{\prime}\right\rangle,\left\langle u, u^{\prime}\right\rangle\right)>0$. Envy-freeness and same-sidedness imply for all $i \in N$,

$$
\varphi_{i}\left(N \cup N^{\prime},\left\langle e, e^{\prime}\right\rangle,\left\langle u, u^{\prime}\right\rangle\right)=\varphi_{i+n}\left(N \cup N^{\prime},\left\langle e, e^{\prime}\right\rangle,\left\langle u, u^{\prime}\right\rangle\right) \leq \hat{u}_{i}=\hat{u}_{i+n}^{\prime} .
$$

Population monotonicity, same-sidedness and feasibility imply for all $i \in N$,

$$
\varphi_{i}\left(N \cup N^{\prime},\left\langle e, e^{\prime}\right\rangle,\left\langle u, u^{\prime}\right\rangle\right)=\varphi_{i}(N, e, u)
$$

Similarly for all $i \in N^{\prime}$ it follows that

$$
\varphi_{i}\left(N \cup N^{\prime},\left\langle e, e^{\prime}\right\rangle,\left\langle u, u^{\prime}\right\rangle\right)=\varphi_{i}\left(N^{\prime}, e^{\prime}, u^{\prime}\right)
$$

Hence, for $i \in N, \varphi_{i}(N, e, u)=\varphi_{i+n}\left(N^{\prime}, e^{\prime}, u^{\prime}\right)$. 


\section{Monotonicity}

This section provides a characterization of the uniform reallocation rule which is based on an endowment monotonicity property. Endowment monotonicity means that if, in case of excess demand, the individual endowments decrease (or increase in case of excess supply), then no individual is better off after the change. The characterization says that the uniform reallocation rule is the only rule which is endowment monotonic and envy-free.

The stages of the proof of this characterization are as follows. First it is shown that endowment monotonicity and Pareto optimality imply coordinatewise continuity. Then another preliminary result is obtained. It says that endowment monotonic and envy-free rules have the dummy property. This latter condition means that non-traders are left on their endowments, hence receive zero allotment change. Next we show that endowment monotonic rules which satisfy the dummy property, assign allotments somewhere between the individual endowments and peaks. With these results the characterization follows easily.

Let $\varphi$ be a rule. Because the properties, dealt with in this section, leave the group size and utilities unchanged, we fix the set of agents at $N$ and the profile of utility functions at $u$. Moreover, a problem $\langle N, e, u\rangle$ is now denoted by $e$. Let $x$ and $y$ be two vectors in $I R_{+}^{N}$. Then $x \geq y$ means that $x_{i} \geq y_{i}$ for all $i \in N$.

We say that the pre-rule $\varphi$ is endowment monotonic or monotonic, if for all problems $e$ and $e^{\prime}$ such that $e \leq e^{\prime}$,

$$
\begin{aligned}
& \text { if } z\left(e^{\prime}\right) \geq 0, \text { then } u_{i}\left(\varphi_{i}(e)\right) \leq u_{i}\left(\varphi_{i}\left(e^{\prime}\right)\right) \text { for all } i \in N, \text { and } \\
& \text { if } z(e) \leq 0 \text {, then } u_{i}\left(\varphi_{i}\left(e^{\prime}\right)\right) \leq u_{i}\left(\varphi_{i}(e)\right) \text { for all } i \in N .
\end{aligned}
$$

In [10], Thomson introduced endowment monotonicity properties for division problems. His one-sided resource-monotonicity corresponds to our monotonicity property.

Under same-sidedness monotonicity is equivalent to $\varphi_{i}(e) \leq \varphi_{i}\left(e^{\prime}\right)$ for all $i \in N$ and all $e \leq e^{\prime}$ in $\mathbb{R}_{+}^{N}$ such that $z\left(e^{\prime}\right) \geq 0$ or $z(e) \leq 0$. By this it follows easily that $U^{r}$ is monotonic.

For all $e \in \mathbb{R}_{+}^{N}, \alpha \in \mathbb{R}_{+}$and $i \in N$, let $e(\alpha, i)$ denote a vector of endowments such that $e(\alpha, i)_{k}=e_{k}$ if $k \in N-\{i\}$ and $e(\alpha, i)_{i}=\alpha$. So, $e(\alpha, i)$ is a unilateral change of $e$ by agent $i$. Furthermore, $\alpha$ denotes $i$ 's endowment in that change.

The pre-rule $\varphi$ is said to be coordinatewise continuous if for all $i \in N$ and all $e \in \mathbb{R}_{+}^{N}$ the function $\alpha \longmapsto \varphi_{i}(e(\alpha, i))$ is continuous.

The following lemma says that monotonic rules are coordinatewise continuous. A similar result for allocation problems, without initial endowments, can be found in Thomson [10] (in the proof of Theorem 2). 
Lemma 4.1. Let $\varphi$ be a monotonic rule. Then $\varphi$ is coordinatewise continuous.

Proof. Let $\alpha^{1}, \alpha^{2}, \ldots, \alpha^{t}, \ldots$ be a sequence in $I R_{+}$converging to $\alpha \in \mathbb{R}_{+}$, and let $x:=\varphi(e(\alpha, i))$ and $x^{t}:=\varphi\left(e\left(\alpha^{t}, i\right)\right)$ for some fixed $i \in N$ and all $t \in I N$. We want to show that $x^{t}$ converges to $x$. Without loss of generality suppose that $\alpha^{1}<\alpha^{2}<\alpha^{3}<\ldots<\alpha$. We distinguish two cases.

Case 1: $z(e(\alpha, i)) \geq 0$.

Then $z\left(e\left(\alpha^{t}, i\right)\right)>0$ for all $t \in \mathbb{I N}$. Hence, by same-sidedness, it follows that $x \leq \hat{u}$ and $x^{t} \leq \hat{u}$ for all $t \in \mathbb{I N}$. Now, by monotonicity, $x^{t} \leq x$ for all $t \in I N$ and the sequence $x^{1}, x^{2}, \ldots, x^{t}, \ldots$ is non-decreasing. By feasibility, it follows that

$$
\begin{aligned}
\sum_{j \in N} x_{j}^{t} & =\sum_{j \in N} e\left(\alpha^{t}, i\right)_{j} \text { and } \\
\sum_{j \in N} x_{j} & =\sum_{j \in N} e(\alpha, i)_{j} .
\end{aligned}
$$

Because $e\left(\alpha^{t}, i\right)$ converges to $e(\alpha, i), x^{t}$ converges to $x$.

Case 2: $z(e(\alpha, i))<0$.

Then there is a number $t_{0}$ such that $z\left(e\left(\alpha^{t}, i\right)\right)<0$ for all $t \geq t_{0}$. Without loss of generality let $t_{0}=1$. The proof proceeds similar to Case 1 .

A pre-rule $\varphi$ is said to have the dummy property, if for all non-traders $j$ at problem $e$ the allotment change is zero, i.e., $\varphi_{j}(e)=e_{j}$.

Lemma 4.2 . Let $\varphi$ be a monotonic and envy-free rule. Then $\varphi$ has the dummy property.

Proof. Suppose at problem $e, \varphi_{j}(e) \neq e_{j}$ for some non-trader $j$. This implies that $z(e) \neq 0$ because otherwise, by Pareto optimality, every agent gets his peak. We assume $z(e)>0$, the other case is similar.

By same-sidedness $\varphi_{j}(e)<\widehat{u}_{j}=e_{j}$. Furthermore, by monotonicity it is without loss of generality (lower the endowments if necessary) to assume that all agents, except agent $j$, have either maximal demand or zero as initial endowment, i.e., for all $i \in N-\{j\}, e_{i}=\max \left\{\hat{u}_{i}-m, 0\right\}$, where $m:=\max _{i \neq j}\left\{\hat{u}_{i}-e_{i}\right\}$. Here, $m>0$ because $z(e)>0$. Let $\alpha:=\triangle \varphi_{j}(e)<0$ denote the allotment change of agent $j$. For $0 \leq \varepsilon \leq-\alpha$ we consider the following endowment vector:

$$
\tilde{e}(\varepsilon)_{i}:= \begin{cases}e_{i} & \text { if } i \neq j \\ \varphi_{j}(e)+\varepsilon & \text { if } i=j .\end{cases}
$$

Hence, $\varphi_{j}(e) \leq \tilde{e}(\varepsilon)_{j} \leq e_{j}$. Furthermore, denote the allotment changes at $\tilde{e}(\varepsilon)$ by $\alpha_{i}^{\varepsilon}:=\triangle \varphi_{i}(\tilde{e}(\varepsilon))$ for all $i \in N$. By envy-freeness between $j$ and agents $i \in N-\{j\}$ and monotonicity it follows that, for all $i \neq j$ and $0 \leq \varepsilon \leq-\alpha$ :

$$
\tilde{e}(\varepsilon)_{j}+\alpha_{i}^{\varepsilon}>\hat{u}_{j} \text { or } \tilde{e}(\varepsilon)_{j}+\alpha_{i}^{\varepsilon} \leq \tilde{e}(\varepsilon)_{j}+\alpha_{j}^{\varepsilon}=\varphi_{j}(\widetilde{e}(\varepsilon)) \leq \varphi_{j}(e) \text {. }
$$


Because $\hat{u}_{j}-\tilde{e}(\varepsilon)_{j}=-\alpha-\varepsilon$ this implies for all $i \neq j$ :

$$
\alpha_{i}^{\varepsilon}>-\alpha-\varepsilon \text { or } \alpha_{i}^{\varepsilon} \leq \alpha_{j}^{\varepsilon} \leq \varphi_{j}(e)-\widetilde{e}(\varepsilon)_{j}<0 \text { for all } 0<\varepsilon \leq-\alpha .
$$

Now we prove that $\alpha_{i}^{\varepsilon} \geq 0$ for all $i \neq j$ and $0<\varepsilon \leq-\alpha$. Suppose, to the contrary, that $\alpha_{i}^{\varepsilon}<0$ for some $i \neq j$ and $0<\varepsilon \leq-\alpha$. Then $\widetilde{e}(\varepsilon)_{i}=e_{i}>0$. So $\widehat{u}_{i}-e_{i}=m \geq \widehat{u}_{k}-e_{k}$ for all $k \neq j$. So, because by same-sidedness $\widehat{u}_{k} \geq \alpha_{k}^{\varepsilon}+\widetilde{e}(\varepsilon)_{k}=\alpha_{k}^{\varepsilon}+e_{k}$, it follows that $\widehat{u}_{i} \geq \alpha_{k}^{\varepsilon}+\widetilde{e}(\varepsilon)_{i}$ for all $k \neq j$. Because $i$ does not envy $k$, we must have $\alpha_{k}^{\varepsilon} \leq \alpha_{i}^{\varepsilon}<0$ for all $k \neq j$. Hence, $\alpha_{k}^{\varepsilon}<0$ for all $k \in N$. This, however, contradicts feasibility. So, $\alpha_{k}^{\varepsilon} \geq 0$ for all $k \neq j$, and (3) implies:

$$
\alpha_{i}^{\varepsilon}>-\alpha-\varepsilon \text { for all } i \neq j \text { and all } 0<\varepsilon \leq-\alpha \text {. }
$$

By monotonicity we have for all $0 \leq \varepsilon \leq \tau \leq-\alpha$ and all $i \neq j$ :

$$
\alpha_{i}^{\tau} \geq \alpha_{i}^{\varepsilon} \text {. }
$$

Now (5) and (4) together imply:

$$
\alpha_{i}^{\varepsilon} \geq-\alpha \text { for all } i \neq j \text { and all } 0<\varepsilon \leq-\alpha .
$$

By coordinatewise continuity (Lemma 4.1) (6) implies $\alpha_{i}^{0} \geq-\alpha$. This is only possible if $N=\{i, j\}, \alpha_{i}^{0}=-\alpha$ and $\alpha_{j}^{0}=\alpha$. Hence, $j$ envies $i$.

A pre-rule $\varphi$ is bounded by endowments and peaks, if for all problems $e$ and all $i \in N$

$$
\text { either } e_{i} \leq \varphi_{i}(e) \leq \hat{u}_{i} \text { or } \hat{u}_{i} \leq \varphi_{i}(e) \leq e_{i} \text {. }
$$

Boundedness by endowments and peaks implies the dummy property. Furthermore, boundedness by endowments and peaks implies individual rationality, i.e., for all problems $e$ and all $i \in N$

$$
u_{i}\left(e_{i}\right) \leq u_{i}\left(\varphi_{i}(e)\right) .
$$

The following lemma says that under monotonicity and same-sidedness the dummy property is equivalent to boundedness by endowments and peaks.

Lemma 4.3. Let $\varphi$ be a monotonic rule which has the dummy property. Then $\varphi$ is bounded by endowments and peaks.

Proof. Let $e$ be a problem. Without loss of generality suppose $z(e)>0$. By same-sidedness, $\varphi_{i}(e) \leq \hat{u}_{i}$ for all $i \in N$. Suppose $j \in N$, such that $\varphi_{j}(e)<\hat{u}_{j}$. It suffices to prove that $e_{j} \leq \varphi_{j}(e)$. Consider $\bar{e} \in \mathbb{R}_{+}^{N}$, with $\bar{e}_{i}=e_{i}$ for all $i \in D(e)$ and $\bar{e}_{i}=\hat{u}_{i}$ for all $i \notin D(e)$. By monotonicity, $\varphi_{i}(\bar{e}) \leq \varphi_{i}(e)$ for all $i \in N$. By the dummy property, $\varphi_{i}(\bar{e})=\hat{u}_{i}$ for all $i \notin D(\bar{e})$. Therefore $j \in D(e)=D(\bar{e})$. By monotonicity it is sufficient to prove that $e_{j}=\bar{e}_{j} \leq \varphi_{j}(\bar{e})$. Suppose $\varphi_{j}(\bar{e})<\bar{e}_{j}$. 
Consider $\tilde{e} \in \mathbb{R}_{+}^{N}$, such that $\tilde{e}_{i}=\bar{e}_{i}$ for all $i \in N-\{j\}$ and $\tilde{e}_{j}=\hat{u}_{j}$. Because $\varphi_{j}(\bar{e})<\bar{e}_{j}$, it follows by same-sidedness and feasibility that $D(\bar{e}) \neq$ $\{j\}$. Hence $z(\tilde{e})>0$. Therefore by monotonicity we have

$$
\varphi_{i}(\bar{e}) \leq \varphi_{i}(\tilde{e}) \text { for all } i \in N .
$$

By feasibility this yields $\varphi_{j}(\hat{e}) \leq \varphi_{j}(\bar{e})+\hat{u}_{j}-\bar{e}_{j}<\hat{u}_{j}=\hat{e}_{j}$. This, however, contradicts the dummy property. So, $\varphi_{j}(\bar{e}) \geq \bar{e}_{j}$.

Finally, we can prove the characterization of this section.

Theorem 4.4. The uniform reallocation rule is the only rule which is envyfree and monotonic.

Proof. The uniform reallocation rule satisfies both properties. In order to prove that it is the only one let $\varphi$ be a rule with these properties, and consider problem $e$. Without loss of generality suppose $z(e)>0$.

By same-sidedness and boundedness by endowments and peaks (Lemmas 4.2 and 4.3$)$ it follows that $\varphi_{i}(e)=\hat{u}_{i}$ for all $i \notin D(e)$. By Lemma 2.1 it is sufficient to prove that non-satiated agents get maximal allotment change. Let $i, j \in D(e)$, such that $\varphi_{i}(e)<\hat{u}_{i}$. Then we have to show that $\triangle \varphi_{j}(e) \leq \triangle \varphi_{i}(e)$.

Suppose $\triangle \varphi_{i}(e)<\triangle \varphi_{j}(e)$. Because of envy-freeness, $e_{i}+\triangle \varphi_{j}(e)>\hat{u}_{i}$. Take $k \in S(e)$. Consider $e(\alpha, k), \alpha \leq e_{k}$ and $\alpha \longrightarrow \hat{u}_{k}$. By coordinatewise continuity, monotonicity and envy-freeness it follows that

$$
\epsilon\left(\alpha^{\prime}, k\right)_{i}+\triangle \varphi_{j}\left(e\left(\alpha^{\prime}, k\right)\right)>\hat{u}_{i} \text {, where } \alpha^{\prime}=\hat{u}_{k} .
$$

Note that $k$ is a non-trader at problem $e\left(\alpha^{\prime}, k\right)$. Hence, the set of suppliers has been decreased by one. Repeating this process yields a problem, say $\tilde{e}$, such that $\tilde{e}_{l}=e_{l}$ for all $l \in D(e), \tilde{e}_{k}=\hat{u}_{k}$ for all $k \notin D(e)$ and

$$
\tilde{e}_{i}+\triangle \varphi_{j}(\tilde{e})=e_{i}+\triangle \varphi_{j}(\tilde{e})>\hat{u}_{i} .
$$

In particular, we have $\triangle \varphi_{j}(\hat{e})>0$. But then either feasibility or boundedness by endowments and peaks is violated.

Results on endowment monotonicity for allocation rules can be found in Thomson [10]. There, a characterization of the uniform allocation rule for a restricted class of single-peaked preferences ${ }^{2}$ by one-sided resourcemonotonicity and envy-freeness is derived. Theorem 4.4, which can be seen as an extension of this result to the reallocation case, is based on a different proof technique, and holds for the whole domain of single-peaked preferences.

\footnotetext{
${ }^{2}$ The function $r: \mathbb{R}_{+} \longrightarrow \mathbb{R}_{+} \cup\{\infty\}$ which assigns to each point either the corresponding indifference point on the other side of the peak, and zero or infinity if such a indifference point does not exists, has to be bounded.
} 


\section{Consistency}

In this section two characterizations of the uniform reallocation rule are discussed. Consistency means that under the mechanism at hand subgroups of agents do not redistribute their subtotal differently. So, if a group of agents leaves with their allotments, then, loosely speaking, applying the mechanism on the remaining agents yields the same outcome as before. Actually, our characterizations only involve bilateral consistency, which means that only situations where two agents remain are considered. First we prove that the uniform reallocation rule is the only rule which is bilateral consistent and bounded by endowments and peaks. Then we show that replacing the latter condition by the weaker individual rationality condition, yields a second characterization of the uniform reallocation rule. Finally we show that for problems with at least four agents, bilateral consistency and envyfreeness determine the uniform reallocation rule.

A pre-rule $\varphi$ is said to be bilaterally consistent, if for all problems $\langle N, e, u\rangle$ and all agents $i, j \in N, i \neq j$,

$$
\varphi_{i}\left(\{i, j\}, e(i, j), u_{\mid\{i, j\}}\right)=\varphi_{i}(N, e, u) .
$$

Here, $u_{\mid\{i, j\}}=\langle u(i), u(j)\rangle$ denotes the restriction of $u$ to $\{i, j\}$ and the adjusted endowment vector $e(i, j) \in \mathbb{R}_{+}^{\{i, j\}}$ is defined as follows. Without loss of generality suppose $\Delta \varphi_{i}(N, e, u) \leq \triangle \varphi_{j}(N, e, u)$. Then,

$$
\begin{aligned}
e(i, j)_{j} & :=\max \left\{0, e_{j}+\frac{1}{2}\left(\triangle \varphi_{i}(N, e, u)+\triangle \varphi_{j}(N, e, u)\right)\right\} \text { and } \\
e(i, j)_{i} & :=e_{i}+\left(\triangle \varphi_{i}(N, e, u)+\triangle \varphi_{j}(N, e, u)\right)-\left(e(i, j)_{j}-e_{j}\right) .
\end{aligned}
$$

So, endowment adjustments are as close as possible ${ }^{3}$ to the mean allotment changes of $i$ and $j$. It is straightforward to prove that the adjusted endowments $e(i, j)_{j}$ and $e(i, j)_{i}$ are non-negative. Furthermore, if $\Delta \varphi_{i}(N, e, u) \geq$ 0 , we obtain mean allotment changes

$$
\begin{aligned}
e(i, j)_{j} & :=e_{j}+\frac{1}{2}\left(\triangle \varphi_{i}(N, e, u)+\triangle \varphi_{j}(N, e, u)\right) \text { and } \\
e(i, j)_{i} & :=e_{i}+\frac{1}{2}\left(\triangle \varphi_{i}(N, e, u)+\triangle \varphi_{j}(N, e, u)\right) .
\end{aligned}
$$

The bilateral consistency property for reallocation problems, is based on Thomson's bilateral consistency for allocation problems, see [9]. There, bilateral consistency is defined with respect to the remaining total endowment, which is left after the departure of all except two agents with their allotments. Then, dividing the remaining (total) endowment among the two agents, applying the same allocation rule, yields the same outcome as before.

\footnotetext{
${ }^{3}$ By just applying mean allotment changes negative endowments, which are not admissable in this model, might occur.
} 
In the reallocation case, however, we first have to distribute the leftover among the two agents to get a reduced reallocation problem. So, bilateral consistency in this setting means that if two agents have to redivide their allotments according to the same rule, then this redivision is equal to the original outcome, provided that they start from adjusted endowments.

It is straightforward to prove that the uniform reallocation rule is bilaterally consistent.

The next theorem characterizes this rule as described before.

Theorem 5.1. The uniform reallocation rule is the only rule which is bilateral consistent and bounded by endowments and peaks.

Proof. Let $\varphi$ be such a rule and $\langle N, e, u\rangle$ a problem. It is sufficient to prove that $\varphi(N, e, u)=U^{r}(N, e, u)$. Without loss of generality suppose $z(N, e, u)>0$; the case of excess supply or balancedness is similar. By boundedness by endowments and peaks and same-sidedness, $\varphi_{i}(N, e, u)=\hat{\hat{u}}_{i}$ for all non-demanders $i \notin D(N, e, u)$. Now, by Lemma 2.1 , it is sufficient to show that non-satiated demanders get maximal allotment changes. Let $i, j \in$ $D(N, e, u)$ such that $\varphi_{i}(N, e, u)<\hat{u}_{i}$. We have to show that $\triangle \varphi_{i}(N, e, u) \geq$ $\triangle \varphi_{j}(N, e, u)$.

To the contrary, suppose

$$
\triangle \varphi_{j}(N, e, u)>\triangle \varphi_{i}(N, e, u) .
$$

Because of boundedness by endowments and peaks $\triangle \varphi_{i}(N, e, u) \geq 0$. So, $e(i, j)_{j} \neq 0$. Consider problem $\left\langle\{i, j\}, e(i, j), u_{\mid\{i, j\}}\right\rangle$. By bilateral consistency,

$$
\varphi_{i}\left(\{i, j\}, e(i, j), u_{\mid\{i, j\}}\right)=\varphi_{i}(N, e, u) .
$$

Because $e(i, j)_{i}=e_{i}+\frac{1}{2}\left(\triangle \varphi_{i}(N, e, u)+\triangle \varphi_{j}(N, e, u)\right)$ and (7) we have

$$
\varphi_{i}(N, e, u)<e(i, j)_{i} .
$$

If $e(i, j)_{i} \leq \hat{u}_{i}$, then obviously boundedness by endowments and peaks is violated. If $e(i, j)_{i}>\hat{u}_{i}$, then by boundedness by endowments and peaks

$$
\varphi_{i}\left(\{i, j\}, e(i, j), u_{\mid\{i, j\}}\right) \geq \hat{u}_{i} .
$$

But as $\varphi_{i}\left(\{i, j\}, \epsilon(i, j), u_{\mid\{i, j\}}\right)=\varphi_{i}(N, e, u)<\hat{u}_{i}$, this cannot be the case. Hence, we have a contradiction and are done.

Note that Theorem 5.1 holds also if we fix $N$.

The following theorem shows that under Pareto optimality and bilateral consistency the boundedness condition of the previous theorem and individual rationality are equivalent if there are at least three agents.

Theorem 5.2。 For problems with at least three agents, the uniform reallocation rule is the only individually rational and bilaterally consistent rule. 
Proof. Let $\varphi$ be an individually rational and bilaterally consistent rule. It is sufficient to prove that $\varphi$ is bounded by endowments and peaks (Theorem 5.1). Let $\langle N, e, u\rangle$ be a problem. Without loss of generality suppose $z(N, e, u)>0$; the excess supply or balancedness case is similar. By individual rationality and same-sidedness,

$$
e_{j} \leq \varphi_{j}(N, e, u) \leq \hat{u}_{j} \text { for all } j \notin S(N, e, u) .
$$

Take a supplier $i \in S(N, e, u)$, then by same-sidedness $\varphi_{i}(N, e, u) \leq \hat{u}_{i}$. Now suppose that $\varphi_{i}(N, e, u)<\hat{u}_{i}$. We deduce a contradiction and are done.

By feasibility and individual rationality there is a demander $j \in D(N, e, u)$ such that

$$
\triangle \varphi_{j}(N, e, u)>0
$$

By bilateral consistency

$$
\varphi_{i}(N, e, u)=\varphi_{i}\left(\{i, j\}, e(i, j), u_{\mid\{i, j\}}\right) .
$$

It follows from (8) that $e(i, j)_{i}>\varphi_{i}(N, e, u)$. So, by individual rationality, $(9)$, and $\widehat{u}_{i}>\varphi_{i}(N, e, u)$,

$$
e(i, j)_{i} \geq \hat{u}_{i}>\varphi_{i}(N, e, u) .
$$

Let $M=\{i, j, l\}$, where $l \notin\{i, j\}$. Take $\bar{e} \in \mathbb{R}_{+}^{N}$ such that $\bar{e}_{k}=e(i, j)_{k}$ for $k \in\{i, j\}$ and $\bar{e}_{l}=e(i, j)_{i}$. Take $u^{\prime} \in \mathcal{U}^{M}$ such that $u_{\mid\{i, j\}}^{\prime}=u_{\mid\{i, j\}}$ and $\hat{u}_{l}^{\prime}=\bar{e}_{l}$. So, the problem $\left\langle\{i, j\}, e(i, j), u_{\mid\{i, j\}}\right\rangle$ is enlarged with a non-trader $l$ and this yields $\left\langle M, \bar{e}, u^{\prime}\right\rangle$. By individual rationality $\varphi_{l}\left(M, \bar{e}, u^{\prime}\right)=\hat{u}_{l}^{\prime}=\bar{e}_{l}$. So, $\bar{e}(i, j)=e(i, j)$. Because $u_{\{\{i, j\}}^{\prime}=u_{\mid\{i, j\}}$, by bilateral consistency and $(9)$,

$$
\varphi_{k}\left(M, \bar{e}, u^{\prime}\right)=\varphi_{k}\left(\{i, j\}, e(i, j), u_{\mid\{i, j\}}\right)=\varphi_{k}(N, e, u) \text { for } k \in\{i, j\} .
$$

Hence, by bilateral consistency we have for $k \in\{i, l\}$

$$
\varphi_{k}\left(M, \bar{e}, u^{\prime}\right)=\varphi_{k}\left(\{i, l\}, \bar{e}(i, l), u_{\mid\{i, l\}}^{\prime}\right) .
$$

Since $\triangle \varphi_{l}\left(M, \bar{e}, u^{\prime}\right)=0$ and because of the choice of $\bar{e}_{l}$ and (10)

$$
\begin{aligned}
\bar{e}(i, l)_{i} & =\bar{e}_{i}+\frac{1}{2}\left(\triangle \varphi_{i}\left(M, \bar{e}, u^{\prime}\right)+\triangle \varphi_{l}\left(M, \bar{e}, u^{\prime}\right)\right) \\
& =\bar{e}_{i}+\frac{1}{2} \triangle \varphi_{i}\left(M, \bar{e}, u^{\prime}\right) \\
& =\bar{e}_{i}+\frac{1}{2}\left(\varphi_{i}\left(M, \bar{e}, u^{\prime}\right)-\bar{e}_{i}\right) \\
& =\frac{1}{2} \epsilon(i, j)_{i}+\frac{1}{2} \varphi_{i}(N, e, u)>\varphi_{i}(N, e, u)
\end{aligned}
$$


By individual rationality $\bar{e}(i, l)_{i} \geq \hat{u}_{i}>\varphi_{i}(N, e, u)$. By adding non-traders in this manner we obtain a sequence $\bar{e}^{t}\left(i, l_{t}\right)$ such that

$$
\begin{aligned}
\bar{e}^{t}\left(i, l_{t}\right)_{i} & \geq \hat{u}_{i}>\varphi_{i}(N, e, u) \text { and } \\
\bar{e}^{t}\left(i, l_{t}\right)_{i} & =\left(\frac{1}{2}\right)^{t} e(i, j)_{i}+\sum_{k=1}^{t}\left(\frac{1}{2}\right)^{k} \varphi_{i}(N, e, u) \\
& =\left(\frac{1}{2}\right)^{t} e(i, j)_{i}+\left(1-\left(\frac{1}{2}\right)^{t}\right) \varphi_{i}(N, e, u) \text { for all } t \in I N .
\end{aligned}
$$

This obviously yields a contradiction.

The previous theorem holds if we fix $N$ and if $N$ has at least three agents. For fixed $N$ with two agents the theorem does not hold, because in that situation bilateral consistency has no impact.

The last characterization in this section is obtained by extending a result of Dagan ([4], Lemma 2) to reallocation rules and applying bilateral consistency and Theorem 3.1.

Theorem 5.3. For problems with at least four agents, the uniform reallocation rule is the unique rule satisfying envy-freeness and bilateral consistency.

As already mentioned above, we use in the proof of Theorem 5.3 the following extension of a result of Dagan ([4], Lemma 2) for allocation rules.

Lemma 5.4. Let there be at least four agents. If a rule is bilaterally consistent and envy-free, then the rule satisfies peaks-onliness for all two-person problems.

The proof of Lemma 5.4 is similar to the proof of Dagan's result [4], Lemma 2.

Proof. Let $\varphi$ be a bilaterally consistent and envy-free rule and consider the two-person problem $\langle N, e, u\rangle, N=\{i, j\}$. To show peaks-onliness we have to prove that for utility functions $u, u^{\prime}$ with $\hat{u}=\hat{u}^{\prime}$ it holds that

$$
\varphi(N, e, u)=\varphi\left(N, e, u^{\prime}\right) .
$$

Because there are at least four agents, we can consider the problem $\left\langle N^{\prime}, e, u^{\prime}\right\rangle, N^{\prime}=\{k, l\}$ such that $N \cap N^{\prime}=\emptyset$ and $\hat{u}_{i}=\hat{u}_{k}^{\prime}, \hat{u}_{j}=\hat{u}_{l}^{\prime}$. Merging the two problems yields $\left(N \cup N^{\prime},\langle e, e\rangle,\left\langle u, u^{\prime}\right\rangle\right)$. By Pareto optimality

$$
\begin{aligned}
& \varphi_{m}\left(N \cup N^{\prime},\langle e, e\rangle,\left\langle u, u^{\prime}\right\rangle\right) \leq\left\langle u, u^{\prime}\right\rangle \text { for all } m \in N \cup N^{\prime} \text { or } \\
& \varphi_{m}\left(N \cup N^{\prime},\langle e, e\rangle,\left\langle u, u^{\prime}\right\rangle\right) \geq\left\langle u, u^{\prime}\right\rangle, \text { for all } m \in N \cup N^{\prime} .
\end{aligned}
$$

Then, by envy-freeness

$$
\begin{aligned}
& \varphi_{i}\left(N \cup N^{\prime},\langle e, e\rangle,\left\langle u, u^{\prime}\right\rangle\right)=\varphi_{k}\left(N \cup N^{\prime},\langle e, e\rangle,\left\langle u, u^{\prime}\right\rangle\right) \text { and } \\
& \varphi_{j}\left(N \cup N^{\prime},\langle e, e\rangle,\left\langle u, u^{\prime}\right\rangle\right)=\varphi_{l}\left(N \cup N^{\prime},\langle e, e\rangle,\left\langle u, u^{\prime}\right\rangle\right) .
\end{aligned}
$$


By applying bilateral consistency on $N$ and $N^{\prime}$ as remaining agents we obtain

$$
\begin{aligned}
\varphi_{m}(N, e, u) & =\varphi_{m}\left(N \cup N^{\prime},\langle e, e\rangle,\left\langle u, u^{\prime}\right\rangle\right), m \in N, \text { and } \\
\varphi_{n}\left(N^{\prime}, e, u^{\prime}\right) & =\varphi_{n}\left(N \cup N^{\prime},\langle e, e\rangle,\left\langle u, u^{\prime}\right\rangle\right), n \in N^{\prime} .
\end{aligned}
$$

Equation (11) together with (12) yields

$$
\varphi(N, e, u)=\varphi\left(N^{\prime}, e, u^{\prime}\right) .
$$

Now, by applying a similar argument as above to the problems $\left\langle N^{\prime}, e, u^{\prime}\right\rangle$ and $\left\langle N, e, u^{\prime}\right\rangle$ it follows that

$$
\varphi\left(N^{\prime}, e, u^{\prime}\right)=\varphi\left(N, e, u^{\prime}\right)
$$

Hence, ((13) and (14)) the lemma is proven.

Proof of Theorem 5.3. Let $\varphi$ be a bilaterally consistent and envy-free rule. Then, bilateral consistency together with Lemma 5.4 implies peaks-onliness for problems with an arbitrary number of agents $n \geq 4$. Then, by Theorem 3.1 the rule $\varphi$ equals the uniform reallocation rule for problems with at least four agents.

In Thomson [9] and Dagan [4] results similar to those described in Theorem 5.2 and Theorem 5.3 are given for allocation rules. Thomson includes a continuity condition in his characterizations ([9], Theorem 1, Theorem 2), besides bilateral consistency, individual rationality from equal division or envy-freeness respectively. Dagan proves that the results of Thomson remain true without continuity for allocation problems with at least four agents ([4], Theorem 2, Theorem 3). Now, "translating" the steps of the proofs ${ }^{4}$ in Dagans characterizations into the reallocation setting is almost sufficient to get alternative proofs of Theorem 5.2 and Theorem 5.3. The argument of converse consistency (see [4], Lemma 4), which completes the proofs of the characterizations, however, has no equivalent in the reallocation setting. By assuming that a rule, satisfying the characterizing properties, does not equal the uniform reallocation rule, and using bilateral consistency, a contradiction, which completes the alternative proofs, is easily derived.

\section{Strategy-Proofness}

In this section we discuss characterizations of the uniform reallocation rule in which endowment strategy-proofness plays a prominent role. This condition makes sense in those situations where the initial endowments are private information and the preferences are known. It guarantees, so to speak, that withholding some of the endowment by an agent is not profitable for that agent, whatever the other agents do. So, truth-telling is a weakly dominant strategy. If agents were also allowed to offer more than their actually possession, then feasibility could cause that some agents obtain a negative allocation. Because our model does not allow such assignments,

\footnotetext{
${ }^{4}[4]$, Lemmas $2,3,5$ and 6 can be proved in their "reallocation version".
} 
supplies are considered to be real amounts handed out to the mechanism. Then, demands can be faked only by withholding endowment.

A pre-rule $\varphi$ is said to be (endowment) strategy-proof, if for all problems $\langle N, e, u\rangle$, all agents $i \in N$ and all $e^{\prime} \in \mathbb{R}_{+}^{N}$, with $\epsilon_{j}^{\prime}=e_{j}$ for all $j \in N-\{i\}$ and $e_{i}^{\prime} \leq e_{i}$,

$$
u_{i}\left(\varphi_{i}(N, e, u)\right) \geq u_{i}\left(e_{i}+\Delta \varphi_{i}\left(N, e^{\prime}, u\right)\right) .
$$

If $i$ acts strategically and pretends to have $e_{i}^{\prime}$ instead of $e_{i}$, then the rule $\varphi$ assigns $\varphi_{i}\left(N, e^{\prime}, u\right)$ to $i$. But, as endowments are private information, $\varphi$ can better be interpreted as a reallocation which assigns allotment changes. So, $i$ 's actual allotment in that situation is $e_{i}+\Delta \varphi_{i}\left(N, e^{\prime}, u\right)$. Therefore, strategy-proofness is defined in this way. It means that $i$ cannot envy himself in a situation of withholding endowment.

The following Lemma shows that if a rule is strategy-proof, then withholding endowment by non-satiated agents yields a smaller allotment change, in case of excess demand, and a greater allotment change, in case of excess supply.

Lemma 6.1. Let $\varphi$ be a strategy-proof rule. Let $\langle N, e, u\rangle$ be a problem and $i \in N$ such that $\varphi_{i}(N, e, u) \neq \hat{u}_{i}$. Let $e^{\prime} \in \mathbb{R}_{+}^{N}$ such that $e_{j}=\epsilon_{j}^{\prime}$ for all $j \in N-\{i\}$ and $e_{i} \geq \epsilon_{i}^{\prime}$. Then:

If $z\left(N, e^{\prime}, u\right) \leq 0$, then $\Delta \varphi_{i}\left(N, e^{\prime}, u\right) \geq \Delta \varphi_{i}(N, e, u)$, and if $z(N, e, u)>0$, then $\Delta \varphi_{i}\left(N, e^{\prime}, u\right) \leq \Delta \varphi_{i}(N, e, u)$.

Proof. Suppose $z\left(N, e^{\prime}, u\right) \leq 0$. Then $\varphi_{i}(N, e, u)>\hat{u}_{i}$ by same-sidedness. Let $f:=\min \left\{x \in R_{+} \mid u_{i}(x) \geq u_{i}\left(\varphi_{i}(N, e, u)\right)\right\}$. Obviously, $f<\hat{u}_{i}$. By strategy-proofness it follows that $\varphi_{i}\left(N, \epsilon^{\prime}, u\right)-e_{i}^{\prime}+e_{i} \leq f$ or $\varphi_{i}\left(N, \epsilon^{\prime}, u\right)-$ $e_{i}^{\prime}+e_{i} \geq \varphi_{i}(N, e, u)$. Because $e_{i} \geq e_{i}^{\prime}$ and $f<\hat{u}_{i} \leq \varphi_{i}\left(N, e^{\prime}, u\right)$ by samesidedness, the former cannot be true. The latter implies the desired result.

Suppose $z(N, e, u)>0$. Then $\varphi_{i}(N, e, u)<\hat{u}_{i}$ by same-sidedness. Let $f:=\sup \left\{x \in \mathbb{R}_{+} \mid u_{i}(x) \geq u_{i}\left(\varphi_{i}(N, e, u)\right)\right\}$. Obviously, $f>\hat{u}_{i}$. Suppose $e_{i}-e_{i}^{\prime}<f-\hat{u}_{i}$. By strategy-proofness $\varphi_{i}\left(N, e^{\prime}, u\right)-e_{i}^{\prime}+e_{i} \leq \varphi_{i}(N, e, u)$ or $\varphi_{i}\left(N, e^{\prime}, u\right)-\epsilon_{i}^{\prime}+e_{i} \geq f$. Because $e_{i}-\epsilon_{i}^{\prime}<f-\hat{u}_{i} \leq f-\varphi_{i}\left(N, e^{\prime}, u\right)$, by same-sidedness, it follows that the latter cannot be the case. The first yields the desired result. In case of $e_{i}-\epsilon_{i}^{\prime} \geq f-\hat{u}_{i}$, we shift $e_{i}$ stepwise (with the size of the steps small enough) to $e_{i}^{\prime}$ and apply the same argument as above in each step.

We have the following consequence of the previous lemma.

Corollary 6.2. Let $\varphi$ be a strategy-proof rule. Let $\langle N, e, u\rangle$ be a problem with excess demand. Then,

$$
\begin{array}{ll}
e_{i} \leq \varphi_{i}(N, e, u) \leq \hat{u}_{i} & \text { for all } i \in D(N, e, u), \text { and } \\
\varphi_{j}(N, e, u)=\hat{u}_{j} & \text { for all } j \notin D(N, e, u) .
\end{array}
$$


Proof. By same-sidedness, $\varphi_{k}(N, e, u) \leq \hat{u}_{k}$ for all $k \in N$. For all $k \in N$ let $e(k) \in \mathbb{R}_{+}^{N}$ such that $e(k)_{l}=e_{l}$ for all $l \in N-\{k\}$ and $e(k)_{k}=0$. Take $k \in$ $N$. Suppose $\varphi_{k}(N, e, u)<\hat{u}_{k}$. Then $\triangle \varphi_{k}(N, e(k), u) \leq \triangle \varphi_{k}(N, e, u)$ by Lemma 6.1. Because $\varphi_{k}(N, e(k), u) \geq 0$, it follows that $\triangle \varphi_{k}(N, e(k), u) \geq$ 0 . So, $\triangle \varphi_{k}(N, e, u) \geq 0$. Therefore $e_{k} \leq \varphi_{k}(N, e, u)$. So, $k \in D(N, e, u)$. This completes the proof.

A similar result cannot be obtained for the excess supply case, even if there were an upper bound for the endowments. (For instance, if there are finite resources.) This is due to the asymmetry in the definition, caused by the requirement of $e_{i}^{\prime} \leq e_{i}$. Therefore, strategy-proofness as defined here, has not such a great impact on the solution as one would expect. The following theorem characterizes the uniform reallocation rule as the only strategy-proof and bilaterally consistent rule which has the dummy property. Recalling Theorem 5.2, Theorem 6.3 implies that, if there are at least three agents, under bilateral consistency and Pareto optimality, strategy-proofness together with the dummy property is equivalent to individual rationality.

Theorem 6.3. The uniform reallocation rule is the only rule which is bilaterally consistent, strategy-proof, and has the dummy property.

Proof. In order to prove that $U^{r}$ is the only rule with these properties, let $\varphi$ be such a rule. Let $\langle N, e, u\rangle$ be a problem. If there is excess demand we are done by Corollary 6.2 and Theorem 5.1. For $z(N, e, u)=0$, Pareto optimality implies $\varphi(N, e, u)=U^{r}(N, e, u)$. Therefore suppose $z(N, e, u)<$ 0 . We prove that $\varphi$ is bounded by endowments and peaks at $\langle N, e, u\rangle$. Then in view of Theorem 5.1 we are done.

By same-sidedness for all $k \in N$,

$$
\varphi_{k}(N, e, u) \geq \hat{u}_{k} \text {. }
$$

Let $j \in S(N, e, u)$. Consider $e^{\prime} \in I_{+}^{N}$ such that $e_{k}^{\prime}=e_{k}$ for all $k \in N-\{j\}$ and $e_{j}^{\prime}=\max \left\{\hat{u}_{j}, e_{j}-s(N, e, u)+d(N, e, u)\right\}$. So, at $\left\langle N, e^{\prime}, u\right\rangle$ agent $j$ is either a non-trader or at $e^{\prime}$ demand equals supply. Therefore, $\varphi_{j}\left(N, e^{\prime}, u\right)=$ $\hat{u}_{j}$. So, $\triangle \varphi_{j}\left(N, e^{\prime}, u\right) \leq 0$. By Lemma 6.1 we have

$$
\triangle \varphi_{j}(N, e, u) \leq \triangle \varphi_{j}\left(N, e^{\prime}, u\right) \leq 0 .
$$

So, $e_{j} \geq \varphi_{j}(N, e, u) \geq \hat{u}_{j}$.

Let $j \in D(N, e, u)$. It is sufficient to prove that $\varphi_{j}(N, e, u) \leq \hat{u}_{j}$. Suppose, to the contrary, that $\varphi_{j}(N, e, u)>\hat{u}_{j}$. Then, by feasibility, there is a supplier i. So, $\triangle \varphi_{i}(N, e, u) \leq 0$ by the previous step of the proof. By bilateral consistency

$$
\varphi_{j}\left(\{i, j\}, e(i, j), u_{\mid\{i, j\}}\right)=\varphi_{j}(N, e, u)
$$

Because $\triangle \varphi_{i}(N, e, u) \leq 0, e(i, j)_{j}<\varphi_{j}(N, e, u)$. Above, we proved that suppliers obtain an allotment change between their peak and their endowment. Therefore, at $\left\langle\{i, j\}, e(i, j), u_{\mid\{i, j\}}\right\rangle j$ cannot be a supplier. So, $e(i, j)_{j} \leq \hat{u}_{j}$. Now, similarly as in the proof of Theorem 5.2, by introducing a sequence 
of non-traders $l_{1}, l_{2}, \ldots, l_{t}, \ldots$ we obtain a sequence of endowments $e\left(i, l_{t}\right)_{j}$ converging to $\varphi_{j}\left(\left\{j, l_{t}\right\}, e\left(j, l_{t}\right), u_{\mid\left\{j, l_{t}\right\}}\right)=\varphi_{j}(N, e, u)>\hat{u}_{j}$, where $j$ cannot be a supplier at $\left\langle\left\{j, l_{t}\right\}, e\left(j, l_{t}\right), u_{\mid\left\{j, l_{t}\right\}}\right\rangle$. This cannot be the case.

In the second and last characterization of this section the condition of reversibility is needed.

A pre-rule $\varphi$ is said to be reversible if for all problems $\langle N, e, u\rangle$ and $\left\langle N, e^{\prime}, u^{\prime}\right\rangle$, such that $e_{i}-\hat{u}_{i}=-\left(e_{i}^{\prime}-\hat{u}_{i}\right)$ for all $i \in N$,

$$
\Delta \varphi_{i}(N, e, u)=-\Delta \varphi_{i}\left(N, e^{\prime}, u^{\prime}\right) \text { for all } i \in N .
$$

So, if all agents demand at $\langle N, e, u\rangle$ as much as they supply at $\left\langle N, e^{\prime}, u^{\prime}\right\rangle$, then their allotment change at $\left\langle N, e^{\prime}, u^{\prime}\right\rangle$ is the reversal of that at $\langle N, e, u\rangle$.

Clearly, by applying reversibility two times we obtain that a solution only depends on the demands and the supplies. That is, if $\varphi$ is a reversible rule and $\langle N, e, u\rangle$ and $\left\langle N, \epsilon^{\prime}, u^{\prime}\right\rangle$ are two problems such that $e_{i}-\hat{u}_{i}=\epsilon_{i}^{\prime}-\hat{u}_{i}^{\prime}$ for all $i \in N$, then

$$
\Delta \varphi_{i}(N, e, u)=\Delta \varphi_{i}\left(N, e^{\prime}, u^{\prime}\right) \text { for all } i \in N .
$$

In particular, this means that reversibility implies peaks-onliness.

Furthermore, we need an equal-treatment condition in the following theorem.

A pre-rule $\varphi$ is said to be equally-treating if for all problems $\langle N, e, u\rangle$ and all $i, j \in N$, such that $e_{i}-\hat{u}_{i}=e_{j}-\hat{u}_{j}$,

$$
\Delta \varphi_{i}(N, e, u)=\Delta \varphi_{j}(N, e, u) .
$$

The equal-treatment condition we introduce here is stronger than the equal-treatment condition introduced in Klaus, Peters and Storcken [6] for reallocation rules.

By definition the uniform reallocation rule satisfies reversibility and equaltreatment. The following theorem says that it is the only such rule which in addition is strategy-proof.

Theorem 6.4. The uniform reallocation rule is the only reversible, equallytreating and strategy-proof rule.

Proof. Let $\varphi$ be such a rule. By reversibility it is sufficient to consider only problems with excess demand. Let $\langle N, \bar{e}, u\rangle$ be a problem such that $z(N, \bar{e}, u)>0$. We prove that $\varphi(N, \bar{e}, u)=U^{r}(N, \bar{e}, u)$.

Because $\varphi$ only depends on demands and supplies it is without loss of generality to suppose that $\hat{u}_{i}=\hat{u}_{j}$ for all $i, j \in N$ and $2 \hat{u}_{i}-\bar{e} \geq 0$ for all $i \in N$.

Without loss of generality suppose $D(N, \bar{e}, u)=\{1,2, \ldots, m\}=: M$. Consider $e \in \mathbb{R}_{+}^{N}$ such that $e_{k} \leq \hat{u}_{k}$ for all $k \in M$, and $e_{k}=\bar{e}_{k}$ for all 
$k \in N-M$. Let $\lambda(e)=\left|\left\{k \in M \mid e_{k} \neq 0\right\}\right|$. Let $O(e)=\left\{k \in M \mid e_{k}=0\right\}$. By induction on $t$ we prove that for all such $e$ with $\lambda(e) \leq t$,

$$
\varphi(N, e, u)=U^{r}(N, e, u) .
$$

Clearly, this is sufficient.

Because $N$ and $u$ are fixed, we suppress these symbols from notation in the rest of this proof.

By Corollary 6.2

$$
\varphi_{j}(e)=\hat{u}_{j}=U_{j}^{r}(e) \text { for all } j \notin M .
$$

Basis: $\lambda(e)=0$

Then equal-treatment and (16) yield (15).

Induction step: Let $\lambda(e)=t+1$.

Suppose (15) does not hold. Then, by (16), there is a demander, say $i$, such that $\varphi_{i}(e) \neq U^{r}(e)$. We deduce a contradiction.

By equal-treatment, $U_{k}^{r}(e)=U_{l}^{r}(e)$ and $\varphi_{k}(e)=\varphi_{l}(e)$ for all $k, l \in O(e)$. Therefore, by feasibility, it is without loss of generality to suppose that $i \notin O(e)$. Now there are two cases; both yield a contradiction.

Case 1: $\varphi_{i}(e)<U_{i}^{r}(e)$.

Hence, $\varphi_{i}(e)<\hat{u}_{i}$. Let $e^{\prime} \in \mathbb{R}_{+}^{N}$ such that $e_{k}^{\prime}=e_{k}$ for all $k \in N-\{i\}$ and $e_{i}^{\prime}=0$. By Lemma 6.1, $\triangle \varphi_{i}\left(e^{\prime}\right) \leq \triangle \varphi_{i}(e)$. Because $\triangle U_{i}^{r}\left(e^{\prime}\right) \geq \triangle U_{i}^{r}(e)$, it follows by our induction hypothesis that

$$
\Delta U_{i}^{r}(e)=U_{i}^{r}(e)-e_{i} \leq U_{i}^{r}\left(e^{\prime}\right)=\varphi_{i}\left(e^{\prime}\right) \leq \varphi_{i}(e)-e_{i}=\Delta \varphi_{i}(e),
$$

contradicting our starting point.

Case 2: $\varphi_{i}(e)>U_{i}^{r}(e)$.

Let $e^{\prime}$ be as in Case 1. Then, $U_{i}^{r}(e)<\hat{u}_{i}$. Hence, in this case $\triangle U_{i}^{r}\left(e^{\prime}\right)=$ $\triangle U_{i}^{r}(e)$. Now, it is sufficient to prove that

$$
\triangle \varphi_{i}\left(e^{\prime}\right) \geq \triangle \varphi_{i}(e)
$$

because then, similarly to Case 1 , a contradiction is easily deduced. Let $\bar{e}, \bar{e}^{\prime} \in \mathbb{R}_{+}^{N}$ such that

$$
\hat{u}_{k}-\bar{e}_{k}=-\left(\hat{u}_{k}-e_{k}\right) \text { forall } k \in N
$$

and

$$
\hat{u}_{k}-\bar{e}_{k}^{\prime}=-\left(\hat{u}_{k}-\epsilon_{k}^{\prime}\right) \text { forall } k \in N .
$$

Then, by reversibility, we have

$$
\begin{aligned}
\triangle \varphi_{i}(e) & =-\triangle \varphi_{i}(\bar{e}) \text { and } \\
\triangle \varphi_{i}\left(e^{\prime}\right) & =-\triangle \varphi_{i}\left(\bar{e}^{\prime}\right) .
\end{aligned}
$$

If $\varphi_{i}\left(\bar{e}^{\prime}\right)=\hat{u}_{i}$, then $\varphi_{i}\left(e^{\prime}\right)=\hat{u}_{i}$ and clearly $\triangle \varphi_{i}\left(e^{\prime}\right) \geq \triangle \varphi_{i}(e)$. If $\varphi_{i}\left(\bar{e}^{\prime}\right) \neq \hat{u}_{i}$, then by Lemma 6.1

$$
\triangle \varphi_{i}\left(\bar{e}^{\prime}\right) \leq \triangle \varphi_{i}(\bar{e})
$$

Therefore by (18) and (19) we obtain (17). 


\section{Overview, Sensitivity Analysis, and IndePendence of the CONDITIONS}

In the preceding sections eight characterizations of the uniform reallocation rule were presented. To illustrate the relation between these results, we start this section with a schematic overview of these results. Combining some of the results immediately yields an ninth characterization (Theorem 7.1) by means of bilateral consistency, monotonicity and the dummy property.

Next, we discuss the sensitivity of the results with respect to variations of the model assumptions.

Finally, independence of the conditions in the characterizations is demonstrated.

\section{OVERVIEW OF THE RESUlts}

The following diagram illustrates the logical connections between the results of the foregoing sections.

In the sequel we use the following abbreviations.

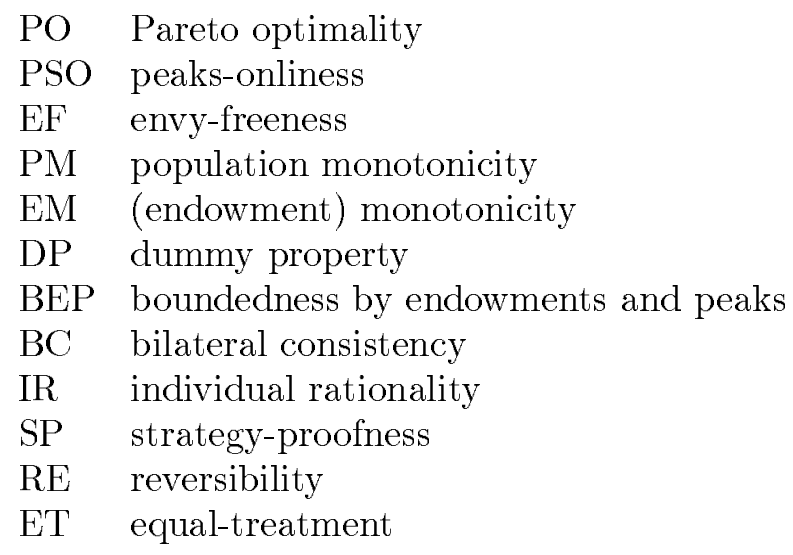

Figure 1 enters here。

Theorem 7.1 (which is added to the diagram) is directly implied by Lemma 4.3 and Theorem 5.1.

Theorem 7.1. The uniform reallocation rule is the only monotonic rule satisfying bilateral consistency and the dummy property.

\section{SENSITIVITy ANALYsis}

The table below indicates the effect of four different model variations on the obtained results. The entries in the cells indicate whether the results remain true. The details are discussed below.

Table 1 enters here。 
(1) In the model presented here, initial endowments and allotments were restricted to non-negative numbers. In other settings one might allow agents to be in debt. In that case, negative endowments and, as a consequence, negative allotments are admitted. Most of the results remain true with little changes in the proofs. In case of bilateral consistency, mean leftover changes for the remaining agents are no longer subject to a non-negativity restriction. The same holds for envy-freeness. This does not affect the proofs. However, the proof of Corollary 6.2 is not valid any more. It is an open question whether Corollary 6.2 or Theorem 6.3 hold in this setting. For Theorem 6.4 there is an alternative proof which is not presented here because of space limitations.

(2) If we suppose that the endowments and the peaks are not only nonnegative but also bounded from above, all results except Theorem 6.4 remain valid. Of course, envy-freeness and bilateral consistency must be adapted to this new situation similarly as in the original model, to guarantee that the (adjusted) endowments, which are used in these conditions, are well-defined. In the proof of Theorem 6.4 we cannot apply reversibility because the reversed problems are not necessarily well-defined in this setting.

(3) Up to now, we assumed that the set of potential agents is infinite. This assumption is crucial for the proof of Theorem 3.2 where we duplicate the number of agents to exploit population monotonicity. It is an open problem whether the characterization of Theorem 3.2 holds for a finite set of potential agents. All other results remain true because the proofs of these theorems apply to a fixed number of agents.

(4) The last model variation we consider concerns the domain of the preferences. We have assumed throughout that the preferences of the agents are single-peaked and continuous. In fact continuity is only needed to prove Lemma 6.1. Whether the characterizations based on this lemma, Theorem 6.3 and Theorem 6.4, hold true for the whole class of single-peaked preferences is not yet clear. All other results remain valid.

\section{INDEPENDENCE OF THE ChaRACTERIZING CONDITIONS}

The logical independence of the characterizing conditions in all theorems discussed in the previous sections and earlier in this section is proven by means of eight reallocation (pre-)rules. These (pre-)rules are defined below.

The endowment pre-rule $\varphi^{e}$ assigns to every individual at every problem $\langle N, e, u\rangle$ the initial endowment:

$$
\varphi^{e}(N, e, u):=e
$$

In case of excess demand (supply), the hierarchical rule $\varphi^{h}$ satiates all suppliers (demanders) and the demanders (suppliers) according to their number. 
So in case of $z(N, e, u) \geq 0$,

$$
\varphi_{i}^{h}(N, e, u):=\hat{u}_{i}
$$

if $i \notin D(N, e, u)$ and

$$
\varphi_{i}^{h}(N, e, u):=\min \left\{\hat{u}_{i}, e_{i}+s(N, e, u)-\sum_{j \in D(N, e, u), j<i} \triangle \varphi_{j}^{h}(N, e, u)\right\}
$$

otherwise. In case of $z(N, e, u) \leq 0, \varphi^{h}$ is defined similarly.

The following maximally satiating rule $\varphi^{\max }$ satiates as many agents as possible. Let $z(N, e, u)>0$ and $D(N, e, u)=\left\{1,2, \ldots, d_{k}\right\}$. Let $d_{1}(N, e, u)=$ $\ldots=d_{t_{1}}(N, e, u)<d_{t_{1}+1}(N, e, u)=\ldots=d_{t_{2}}(N, e, u)<\ldots<d_{t_{r}}(N, e, u)=$ $\ldots=d_{k}(N, e, u)$. Then,

if $i \notin D(N, e, u)$ and

$$
\varphi_{i}^{\max }(N, e, u):=\hat{u}_{i}
$$

$$
\varphi_{i}^{\max }(N, e, u):=\min \left\{\hat{u}_{i}, e_{i}+\frac{1}{t_{s}-t_{s-1}}\left(s(N, e, u)-\sum_{j \leq t_{s-1}} \triangle \varphi_{j}(N, e, u)\right)\right\}
$$

if $i \in D(N, e, u), t_{s-1}<i \leq t_{s}$. Hence, demanders are satiated according to their claims. First minimal demands are satiated uniformly. If there is some supply left, then the next smallest demands are satiated, and so on.

In case of $z(N, e, u)<0, \varphi^{\max }$ is defined similarly.

The following rule is a variation of $\varphi^{\max }$.

$$
\bar{\varphi}^{\max }(N, e, u):= \begin{cases}\varphi^{\max }(N, e, u) & \text { if } z(N, e, u)>0 \text { and } \\ U^{r}(N, e, u) & \text { if } z(N, e, u) \leq 0 .\end{cases}
$$

The rule $\varphi^{\underline{0}}$ is equal to the uniform reallocation rule except for those problems $\langle N, e, u\rangle$ where all peaks are zero. In that case all agents except agent $n$, where $N=\{1, \ldots, n\}$, are satiated and feasibility of the allocation is adjusted on the account of $n$. So, if $\hat{u} \neq \underline{0}$ (the zero vector), then

$$
\varphi^{\underline{0}}(N, e, u):=U^{r}(N, e, u) \text {. }
$$

If $\hat{u}=\underline{0}$, then

$$
\varphi_{i}^{0}(N, e, u):= \begin{cases}0 & \text { for } i<n \text { and } \\ s(N, e, u) & \text { for } i=n .\end{cases}
$$

The following rule $\bar{\varphi}^{\underline{0}}$ is a variation of $\varphi^{\underline{0}}$. Instead of adjusting feasibility on the account of $n$ this is done on the account of the highest numbered supplier. So, if $\hat{u} \neq \underline{0}$, then

$$
\bar{\varphi}^{\underline{0}}(N, e, u):=U^{r}(N, e, u)
$$

For $\hat{u}=\underline{0}$ let $k:=\max \{i \mid i \in S(N, e, u)\}$. Then,

$$
\bar{\varphi}_{i}^{0}(N, e, u):= \begin{cases}0 & \text { for all } i \in N-\{k\} \text { and } \\ s(N, e, u) & \text { for } i=k .\end{cases}
$$


The following rule $\tilde{\varphi}$ is a rule which is not peaks-only. Let $\left\langle N, e^{\prime}, u^{\prime}\right\rangle$ be a problem with $e^{\prime}=\left\langle\frac{5}{2}, 0, \ldots, 0\right\rangle, \hat{u}_{i}^{\prime}=0$ for $i>2$ and $u_{1}^{\prime}=u_{2}^{\prime}$ such that $u_{1}^{\prime}(x)=-\left|\frac{3}{2}-x\right|$ for $x \in[0, \infty)$. Then, $\tilde{\varphi}$ is defined by

$$
\tilde{\varphi}(N, e, u):= \begin{cases}U^{r}(N, e, u) . & \text { if }\langle N, e, u\rangle \neq\left\langle N, e^{\prime}, u^{\prime}\right\rangle \text { and } \\ \left\langle 1, \frac{3}{2}, 0, \ldots, 0\right\rangle & \text { otherwise. }\end{cases}
$$

Finally the rule $\hat{\varphi}$ satiates all agents as much as possible except those with the greatest demand (supply) in case of excess demand (supply). Feasibility is adjusted on their account. If $z(N, e, u)>0$, then

$$
\hat{\varphi}_{i}(N, e, u):= \begin{cases}\hat{u}_{i} & \text { if } i \notin D(N, e, u) \text { and } \\ \min \left\{\hat{u}_{i}, e_{i}+\gamma\right\} & \text { if } i \in D(N, e, u)-G \text { and } \\ e_{i}-\lambda & \text { if } i \in G,\end{cases}
$$

where $G=\arg \max _{i \in D(N, e, u)} d_{i}(N, e, u)$ and $\gamma$ and $\lambda$ are determined by feasibility and the range restriction $e_{i}-\lambda \geq 0(i \in G)$ such that $\gamma$ is maximal and $-\lambda$ is minimal.

In case of $z(N, e, u) \leq 0, \hat{\varphi}$ is defined similarly.

The following table shows which of the previous pre-rules satisfies which of the characterizing conditions. The last nine rows of this table indicate for each characterization and each (pre-)rule which condition is not satisfied by the (pre-)rules while all other characterizing conditions are satisfied.

\section{Table 2 enters here。}

The last table below illustrates the trade-offs between the different characterizations. Roughly speaking there are four groups of conditions; I conditions present in all characterizations (and therefore not interesting with respect to a trade-off discussion), II conditions of equity, III conditions relating different problems, and IV conditions that bound the outcome.

\section{Table 3 enters here.}

Conditions of the first three groups appear in all characterizations. The last group is only present, when bilateral consistency, which also belongs to the second group, is one of the characterizing conditions. Clearly the price which has to be paid for using this hybrid condition of group II and III is either a relatively strong condition of group IV or the weaker dummy property in combination with strategy-proofness or monotonicity. Comparing Theorems 6.3 and 6.4 we see that the trade-off of relaxing the equity condition is compensated by the relatively strong reversibility condition of group IV. 


\section{REFERENCES}

[1] Barbera, S., M.O. Jackson, and A. Neme (1995): "Strategy-Proof Allotment Rules", Working Paper, Universitat Autonoma de Barcelona.

[2] Benassy, J.P. (1982):The Economics of Market Disequilibrium, San Diego: Academic Press.

[3] Ching, S. (1994): "An Alternative Characterization of the Uniform Rule", Social Choice and Welfare, 11, $131-136$.

[4] Dagan, N. (1995): “A Note on Thomson's Characterization of the Uniform Rule”, Working Paper, Hebrew University of Jerusalem (forthcoming in Journal of Economic Theory)

[5] Foley, D. (1967): "Resource Allocation and the Public Sector", Yale Economic Essays, $7,45-98$.

[6] Klaus, B., H. Peters, and T. Storcken ("1995): "Strategy-Proof Division with SinglePeaked Preferences and Initial Endowments", Working Paper, Department of Quantitative Economics, University of Maastricht.

[7] Sprumont, Y. (1991): "The Division Problem with Single-Peaked Preferences: A Characterization of the Uniform Allocation Rule", Econometrica, 59, 509 - 519.

[8] Thomson, W. (1991): "Population-Monotonic Solutions to the Problem of Fair Division when Preferences are Single-Peaked", Economic Theory, forthcoming.

[9] Thomson, W. (1994): "Consistent Solutions to the Problem of Fair Division when Preferences are Single-Peaked", Journal of Economic Theory, 2, 219 - 245.

[10] Thomson, W. (1994): "Resource-Monotonic Solutions to the Problem of Fair Division when Preferences are Single-Peaked", Social Choice and Welfare, 11, $205-223$. 


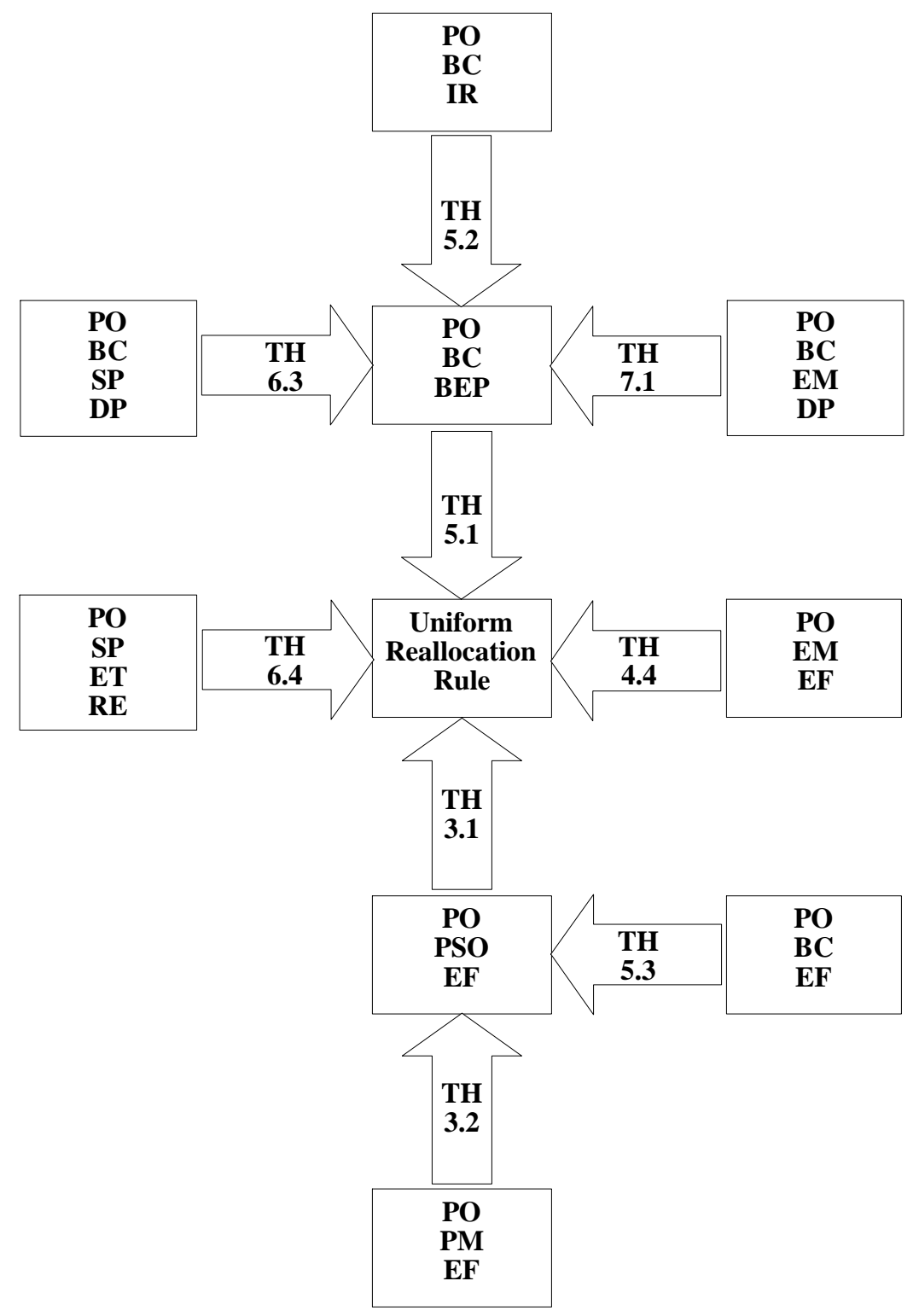

Figure 1 


\begin{tabular}{|c|c|c|c|c|}
\hline & $\begin{array}{c}(1) \\
e \in \mathbb{R}^{N} \\
\text { instead of } \\
e \in \mathbb{R}_{+}^{N}\end{array}$ & $\begin{array}{c}\quad(2) \\
e \in[0, b], \\
b \in \mathbb{R}_{+} \\
\text {instead of } \\
e \in \mathbb{I R}_{+}^{N}\end{array}$ & $\begin{array}{c}(3) \\
N \subseteq\{1, \ldots, m\}, \\
m \geq 3 \\
\text { instead of } \\
N \subset \mathbb{N}\end{array}$ & $\begin{array}{c}(4) \\
u \text { arbitrary } \\
\text { single-peaked } \\
\text { instead of } \\
u \text { single-peaked } \\
\text { and continuous }\end{array}$ \\
\hline $\begin{array}{l}\text { Th } 3.1 \\
\text { PO, PSO, } \\
\text { EF }\end{array}$ & yes & yes & yes & yes \\
\hline $\begin{array}{l}\text { Th } 3.2 \\
\text { PO, PM, } \\
\text { EF }\end{array}$ & yes & yes & $?$ & yes \\
\hline $\begin{array}{l}\text { Th } 4.4 \\
\text { PO, EM, } \\
\text { EF }\end{array}$ & yes & yes & yes & yes \\
\hline $\begin{array}{l}\text { Th } 5.1 \\
\text { PO, BC, } \\
\text { BEP }\end{array}$ & yes & yes & yes & yes \\
\hline $\begin{array}{l}\text { Th } 5.2 \\
\text { PO, BC, } \\
\text { IR }\end{array}$ & yes & yes & yes & yes \\
\hline $\begin{array}{l}\text { Th } 5.3 \\
\text { PO, BC, } \\
\text { EF }\end{array}$ & yes & yes & yes & yes \\
\hline $\begin{array}{l}\text { Th } 6.3 \\
\text { PO, BC, } \\
\text { SP, DP }\end{array}$ & $?$ & yes & yes & $?$ \\
\hline $\begin{array}{l}\text { TH } 6.4 \\
\text { PO, ET, } \\
\text { SP, RE }\end{array}$ & yes & $?$ & yes & $?$ \\
\hline $\begin{array}{l}\text { TH } 7.1 \\
\text { PO, BC, } \\
\text { EM, DP }\end{array}$ & yes & yes & yes & yes \\
\hline
\end{tabular}

TABLE 1 


\begin{tabular}{|c||c|c|c|c|c|c|c|c|}
\hline & $\varphi^{e}$ & $\varphi^{h}$ & $\varphi^{\max }$ & $\bar{\varphi}^{\max }$ & $\varphi^{\underline{0}}$ & $\bar{\varphi}^{\underline{0}}$ & $\tilde{\varphi}$ & $\hat{\varphi}$ \\
\hline \hline PO & N & Y & Y & Y & Y & Y & Y & Y \\
\hline PSO & Y & Y & Y & Y & Y & Y & N & Y \\
\hline EF & Y & N & N & N & N & N & Y & N \\
\hline PM & Y & Y & Y & Y & N & N & N & N \\
\hline EM & Y & Y & N & N & Y & N & N & N \\
\hline BC & Y & N & N & N & Y & N & N & Y \\
\hline BEP & Y & Y & Y & Y & N & N & N & N \\
\hline IR & Y & Y & Y & Y & N & N & Y & N \\
\hline SP & Y & Y & N & Y & Y & N & N & Y \\
\hline DP & Y & Y & Y & Y & N & Y & Y & Y \\
\hline ET & Y & N & Y & Y & N & N & Y & Y \\
\hline RE & Y & Y & Y & N & N & N & N & Y \\
\hline Th 3.1 & PO & EF & EF & EF & EF & EF & PSO & EF \\
\hline Th 3.2 & PO & EF & EF & EF & - & - & PM & - \\
\hline Th 4.4 & PO & EF & EF & EF & EF & EF & EM & - \\
\hline Th 5.1 & PO & BC & BC & BC & BEP & BEP & - & BEP \\
\hline Th 5.2 & PO & BC & BC & BC & IR & IR & BC & IR \\
\hline Th 5.3 & PO & - & - & - & EF & - & BC & EF \\
\hline Th 6.3 & PO & BC & BC & BC & DP & SP & - & - \\
\hline Th 6.4 & PO & ET & SP & RE & - & - & - & - \\
\hline Th 7.1 & PO & BC & BC & BC & DP & - & - & EM \\
\hline
\end{tabular}

Y The rule satisfies the condition in question.

$\mathrm{N}$ The rule does not satisfy the condition in question. 


\begin{tabular}{|c|c|c|c|c|c|c|c|c|c|c|}
\hline & & $\begin{array}{l}\text { Th } \\
3.1\end{array}$ & $\begin{array}{l}\text { Th } \\
3.2\end{array}$ & $\begin{array}{l}\text { Th } \\
4.4\end{array}$ & $\begin{array}{l}\text { Th } \\
5.1\end{array}$ & $\begin{array}{l}\text { Th } \\
5.2\end{array}$ & $\begin{array}{l}\text { Th } \\
5.3\end{array}$ & $\begin{array}{l}\text { Th } \\
6.3\end{array}$ & $\begin{array}{l}\text { Th } \\
6.4\end{array}$ & $\begin{array}{l}\text { Th } \\
7.1\end{array}$ \\
\hline \multirow[t]{2}{*}{$\mathrm{I}$} & $\begin{array}{l}\text { Conditions present in } \\
\text { all characterizations }\end{array}$ & & & & & & & & & \\
\hline & $\mathrm{PO}$ & $x$ & $x$ & $x$ & $x$ & $x$ & $x$ & $x$ & $x$ & $x$ \\
\hline \multirow[t]{6}{*}{ II } & $\begin{array}{l}\text { Conditions relating } \\
\text { different problems }\end{array}$ & & & & & & & & & \\
\hline & $\mathrm{PM}$ & & $x$ & & & & & & & \\
\hline & EM & & & $x$ & & & & & & $x$ \\
\hline & $\mathrm{SP}$ & & & & & & & $x$ & $x$ & \\
\hline & $\mathrm{PSO}$ & $x$ & & & & & & & & \\
\hline & $\mathrm{RE}$ & & & & & & & & $x$ & \\
\hline \multirow[t]{4}{*}{ III } & $\begin{array}{l}\text { Conditions of } \\
\text { equity }\end{array}$ & & & & & & & & & \\
\hline & BC also group II & & & & $x$ & $x$ & $x$ & $x$ & & $x$ \\
\hline & $\mathrm{EF}$ & $x$ & $x$ & $x$ & & & $x$ & & & \\
\hline & $\mathrm{ET}$ & & & & & & & & $x$ & \\
\hline \multirow[t]{4}{*}{ IV } & $\begin{array}{l}\text { Conditions bounding } \\
\text { the outcome }\end{array}$ & & & & & & & & & \\
\hline & IR & & & & & $x$ & & & & \\
\hline & $\mathrm{BEP}$ & & & & $x$ & & & & & \\
\hline & $\mathrm{DP}$ & & & & & & & $x$ & & $x$ \\
\hline
\end{tabular}

$x$ The condition in question (row) is part of the corresponding characterization (column).

TABLE 3

Department of Quantitative Economics, University of Maastricht, P.O. Box 616, 6200 MD MaAstricht, The Netherlands 